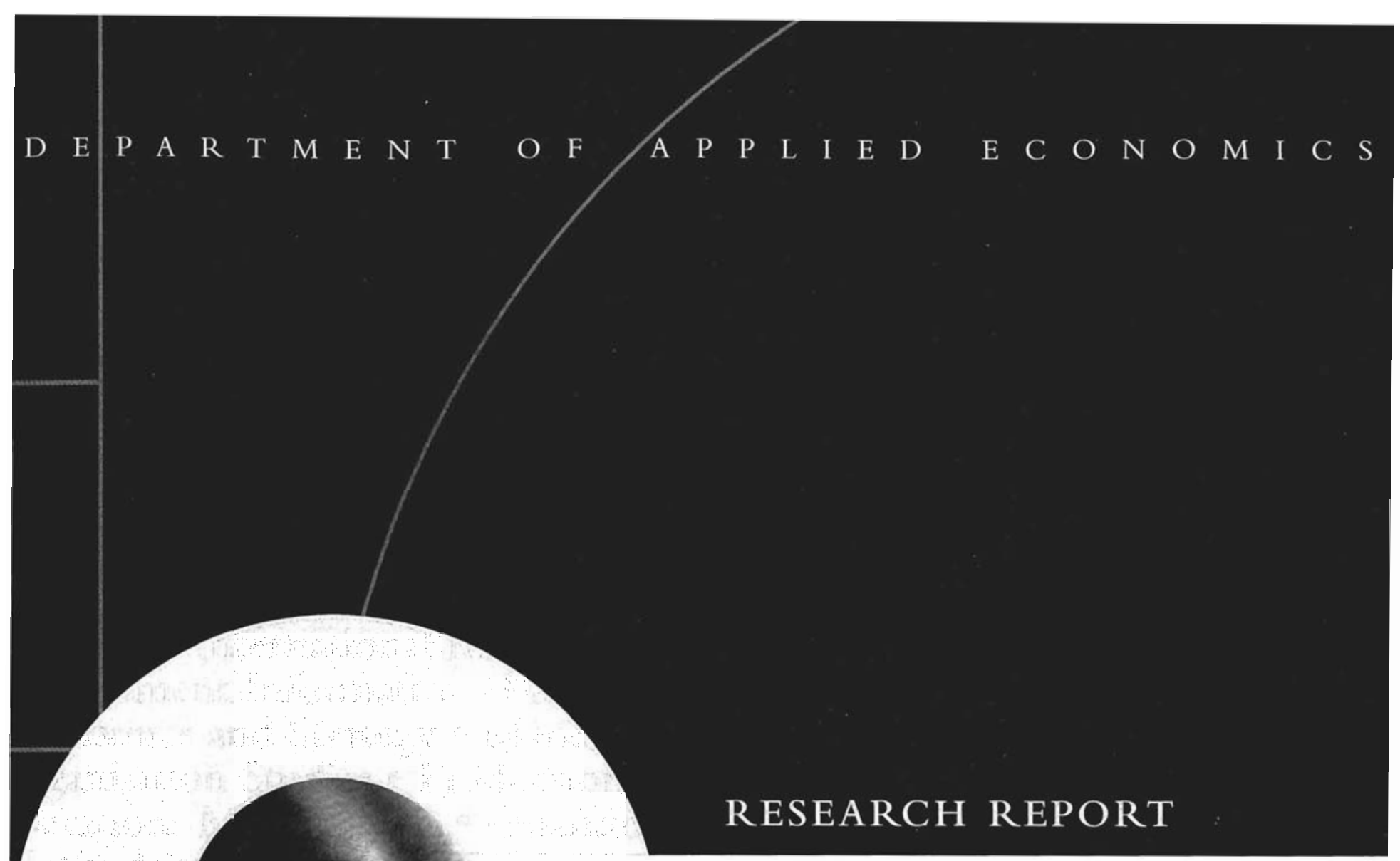

SCHEDULING TRAINEES AT A HOSPITAL DEPARTMENT USING A BRANCH-AND-PRICE APPROACH Jeroen Belien \& Erik Demellemeester

OR 0403 


\title{
Scheduling trainees at a hospital department using a branch-and-price approach
}

\author{
Jeroen Belien ${ }^{1}$, Erik Demeulemeester ${ }^{1}$ \\ 'Department of Applied Economics, K.U.Leuven, Belgium \\ e-mail: < first name>.<family name>@econ.kuleuven.ac.be
}

\begin{abstract}
Scheduling trainees (graduate students) is a complicated problem that has to be solved frequently in many hospital departments. We will describe a trainee scheduling problem encountered in practice (at the ophthalmology department of the university hospital Gasthuisberg, Leuven). In this problem a department has a certain number of trainees at its disposal, which assist specialists in their activities (surgery, consultation, etc.). For each trainee one has to schedule the activities in which (s)he will assist during a certain time horizon, usually one year. Typically, these kind of scheduling problems are characterized by both hard and soft constraints. The hard constraints consist of both work covering constraints and formation requirements, whereas the soft constraints include trainees' preferences and setup restrictions. In this paper we will describe an exact branch-and-price method to solve the problem to optimality.
\end{abstract}

Key words: Staff scheduling, branch-and-price, health care

\section{Introduction}

The problem of scheduling medical trainees to perform a number of activities over a given time horizon is addressed. Recently, a very good bibliographic survey on medical staff rostering problems has appeared (Cheang et al (2002)). Several studies in the literature have utilized mathematical programming techniques to assist in finding efficient staff schedules (see e.g. Warner (1976) or Beaumont (1997)). The main problem of these integer programs lies in the large computation times needed for many practical instances, even to obtain just a feasible solution. To overcome this problem both heuristic approaches and exact approaches that exploit specific features of the problem structure have been developed and described for plenty of staff scheduling applications. The most important heuristic approaches include simulated annealing (e.g. Brusco and Jacobs (1993)), tabu search (e.g. Burke et al. (1998)) and genetic algorithms (e.g. Aickelin and Dowsland (2000)). Regarding exact approaches we can distinguish between constraint programming, branch-and-bound (see e.g. Bosi and Milano (2001)) and branch-and-price approaches. Branch-and-price, a technique in which bounds are calculated by solving the LP relaxation of the problem using a column generation scheme, has gained considerable attention during the last decade. 
Most of the encountered scheduling problems studied in the literature are short-term shift scheduling problems involving some kind of set covering or set partitioning formulation (e.g. Mason and Smith (1998)). Alternatively, 0-1 multi-commodity flow formulations are proposed (e.g. Cappanera and Gallo (2001)). To the best of our knowledge all branch-andprice approaches for staff scheduling problems decompose on staff members, i.e. generate columns per staff member (see e.g. Mehrotra et al (2000)). In contrast, we study a longterm scheduling problem for which we propose a decomposition scheme on the tasks, further referred to as activities, instead of decomposing on the employees. The main advantage of this decomposition strategy is that it results in smaller network problems while pricing out new columns. This approach enables us to find optimal solutions for real-life data sets. The problem will be written as a $0-1$ multi-commodity flow problem with side-constraints, where each activity corresponds to a commodity.

The paper is organized as follows. Firstly, the problem will be stated and written as an integer program. Secondly, to solve the problem to optimality a branch-and-bound algorithm will be elaborated. Next, starting from the drawbacks of the branch-and-bound scheme, a branch-and-price algorithm will be introduced. Thirdly, several features of the branch-and-price scheme are discussed followed by an extensive overview of computational results. Finally, the paper ends with a conclusion and some ideas for future research.

\section{Problem definition}

As the problem originates from a practical context, the exact statement of the problem is not straightforward. The main reason is the presence of both hard and soft constraints. Hard constraints are constraints that cannot be violated, whereas soft constraints are constraints that must be met 'as much as possible'. Firstly, the problem will be described in an informal way by means of an example. Secondly, an integer linear programming (ILP) formulation of the problem will be given.

Consider a hospital department in which trainees have to perform a number of activities over a certain time horizon. Since the trainees have different experience levels, they can be divided in a number of experience groups. Most of the activities include assisting a specialist in a very specific field of the health care. Firstly, for each activity it is known how many trainees of each experience group are required in each period. Secondly, for each trainee it is known which activities (s)he has to perform in order to meet formation objectives. Thirdly, for each trainee it is known for each time period whether or not (s)he is available to be scheduled. Finally, in order to maximize both the efficiency and the quality of the service provided, it is not allowed that activities are split up per trainee. The efficiency decreases with each new activity start of a trainee, because it takes (again) some time to master the skills required for the activity. Moreover, patients have a smaller chance to be treated by one and the same trainee, resulting in less efficient care. In the ideal case each trainee starts each activity only once and performs it for a minimum number of consecutive periods. The last two constraints are soft constraints meaning that they can be violated at a certain 'cost'. Since a split-up in activities is considered to be worse than the violation of a non-availability constraint, we will concentrate on the problem solution in 
which we only relax the non-availability constraints. Therefore, the trainees have to quantify their preferences for having weeks-off. This happens by dividing a number of points per trainee over the scheduling horizon. The higher the number of points a certain period receives, the stronger the trainee feels about not being scheduled during that period.

Let us illustrate this problem with a simple example (see Figure 1). Suppose we have a problem with three activities, four trainees and ten periods. Furthermore, assume that all assistants have the same level of experience. Finally, suppose that each assistant has to perform each activity and this between a minimum of two and maximum of three consecutive periods. This example is graphically represented in Figure 1. The columns represent the trainees and the rows represent the periods. The periods of non-availability for each trainee are marked in grey and the respective 'costs' are indicated. Note that each trainee has divided in total five points over the ten periods. It is realistic that these points are concentrated in a small number of periods.

\begin{tabular}{|c|c|c|c|}
\hline $\operatorname{tr} 1$ & $\operatorname{tr} 2$ & $\operatorname{tr} 3$ & $\operatorname{tr} 4$ \\
\hline & & & \\
\hline 4 & & & \\
\hline & & 4 & \\
\hline & 1 & & \\
\hline & & & \\
\hline & & & 2 \\
\hline 1 & & & \\
\hline & 4 & & \\
\hline & & 1 & \\
\hline & & & 3 \\
\hline
\end{tabular}

\begin{tabular}{l|cccc}
\multicolumn{5}{l}{ min. nr. consecutive periods: } \\
& $\operatorname{tr} 1$ & $\operatorname{tr} 2$ & $\operatorname{tr} 3$ & $\operatorname{tr} 4$ \\
\hline act 1 & 2 & 2 & 2 & 2 \\
act 2 & 2 & 2 & 2 & 2 \\
act 3 & 2 & 2 & 2 & 2
\end{tabular}

\begin{tabular}{l|cccc}
\multicolumn{5}{c}{ max. nr. consecutive periods: } \\
& $\operatorname{tr} 1$ & $\operatorname{tr} 2$ & $\operatorname{tr} 3$ & $\operatorname{tr} 4$ \\
\hline act 1 & 3 & 3 & 3 & 3 \\
act 2 & 3 & 3 & 3 & 3 \\
act 3 & 3 & 3 & 3 & 3
\end{tabular}

Figure 1: Example 1

A possible solution for this problem is represented in figure 2 . In this solution, trainees 1 and 3 are both scheduled during a period in which they actually prefer not to be scheduled, respectively period 7 and period 9 , making up for a total cost of $1+1=2$. In practice, this means that either the trainee has to give up his/her preference for having a period off or the trainee has to be replaced by someone else in this period, resulting in a decrease of the quality of care. As a final remark, observe that in this solution during four time periods no activity is scheduled for particular trainees. During these periods, the trainees will perform activities for which no specific skills are required and for which consequently both experience level and minimal formation requirements are less important. An example of such an activity is consultation. A two-phase approach is thus being used to solve this problem. In the first phase the difficult (hard constrained) activities are scheduled. In the second phase the partial schedule is completed with the easy activities. The scheduling of the easy activities is straightforward and can easily be done manually. Therefore, we will only concentrate on the scheduling of the difficult activities in this paper. 


\begin{tabular}{|c|c|c|c|}
\hline tr 1 & tr 2 & $\operatorname{tr} 3$ & $\operatorname{tr} 4$ \\
\hline & act 1 & act 2 & act 3 \\
\hline 4 & act 1 & act 2 & act 3 \\
\hline act 3 & act 1 & 4 & act 2 \\
\hline act 3 & 1 & act 1 & act 2 \\
\hline & act 3 & act 1 & act 2 \\
\hline act 2 & act 3 & act 1 & 2 \\
\hline act 2 & act 3 & & act 1 \\
\hline act 2 & 4 & act 3 & act 1 \\
\hline act 1 & act 2 & act 3 & \\
\hline act 1 & act 2 & act 3 & 3 \\
\hline
\end{tabular}

\begin{tabular}{l|cccc}
\multicolumn{6}{l}{ min. nr. consecutive periods: } \\
& $\operatorname{tr} 1$ & $\operatorname{tr} 2$ & $\operatorname{tr} 3$ & $\operatorname{tr} 4$ \\
\hline act 1 & 2 & 2 & 2 & 2 \\
act 2 & 2 & 2 & 2 & 2 \\
act 3 & 2 & 2 & 2 & 2
\end{tabular}

max. nr. consecutive periods:

\begin{tabular}{l|cccc} 
& $\operatorname{tr} 1$ & $\operatorname{tr} 2$ & $\operatorname{tr} 3$ & $\operatorname{tr} 4$ \\
\hline act 1 & 3 & 3 & 3 & 3 \\
act 2 & 3 & 3 & 3 & 3 \\
act 3 & 3 & 3 & 3 & 3
\end{tabular}

Figure 2: A solution for example 1

The problem can be mathematically stated as follows. Consider the following binary decision variables:

$$
\begin{aligned}
x_{i j k} & =1 \text { if during period } i \text { trainee } j \text { is scheduled to perform activity } k ; \\
& =0 \text { otherwise. } \\
y_{i j k} & =1 \text { if trainee } j \text { starts activity } k \text { during period } i \\
& =0 \text { otherwise. }
\end{aligned}
$$

Let $p_{i j}$ be the penalty cost charged for assigning trainee $j$ to period $i$. It must be clear that $p_{i j}$ equals 0 if trainee $j$ is available during period $i$. Let $l_{j k}$ and $u_{j k}$ be the respective minimum and maximum number of periods assistant $j$ has to perform activity $k$. Finally, Let $S_{k}$ represent the set of trainees that will perform activity $k$ in the given time horizon (i.e. all trainees $j$ for which $u_{j k}>0$ ). The integer linear programming model (ILP) is given below.

$$
\operatorname{MIN} \sum_{i=1}^{n} \sum_{j=1}^{m} p_{i j} x_{i j k}
$$

\section{S.T.}

1. A trainee can perform no more than one activity per period:

$$
\sum_{k=1}^{p} x_{i j k} \leq 1 \quad \forall i=1, . ., n \text { and } \forall j=1, . ., m
$$

2. At each period every activity has to be performed by exactly one trainee:

$$
\sum_{j \in S_{k}} x_{i j k}=1 \quad \forall i=1, . ., n \text { and } \forall k=1, . ., p
$$

3. Each trainee has to perform each activity between a minimum and maximum number of periods:

$$
\sum_{i=1}^{n} x_{i j k} \geq l_{j k} \quad \forall j=1, . ., m \text { and } \forall k=1, . ., p
$$




$$
\sum_{i=1}^{n} x_{i j k} \geq u_{j k} \quad \forall j=1, . ., m \text { and } \forall k=1, . ., p
$$

4. Each trainee starts each activity only once:

$$
\begin{array}{ll}
y_{1 j k}=x_{1 j k} & \forall j=1, . ., m \text { and } \forall k=1, \ldots, p \\
y_{i j k} \geq x_{i j k}-x_{(i-1) j k} & \forall i=2, . ., n, \forall j=1, . ., m \text { and } \forall k=1, . ., p \\
\sum_{i=1}^{n} y_{i j k} \leq 1 & \forall j=1, . ., m \text { and } \forall k=1, . ., p
\end{array}
$$

5. Integrality constraints:

$$
x_{i j k}, y_{i j k} \in\{0,1\} \quad \forall i=1, . ., n, \forall j=1, . ., m \text { and } \forall k=1, . ., p
$$

For a problem with $n$ time periods, $m$ trainees and $p$ activities, this notation requires $2 n m p$ binary decision variables, a number that is growing rapidly. Even very simple problems require long solution times using this formulation and a commercial ILP solver. Obviously, better formulations and thus smaller solution times could be found by introducing other (integer) decision variables. It seems, however, that a specifically developed solution procedure like branch-and-bound is more suitable to solve this problem.

A last remark concerns activities that require more than one trainee during each period. We replace each of these activities by two or more artificial activities dividing the trainees in the original set $S_{k}$ over these new activities. Consider for instance an activity for which each time period two trainees are required and twelve trainees have to perform the activity. Then, this activity will be replaced by two new activities each of which has to be performed by six trainees. This assumption facilitates the construction of an enumeration scheme at the cost of possibly excluding an optimal solution, since we only consider one particular division of trainees over the newly introduced activities.

In the remainder of this paper an effort will be made to solve the problem to optimality. To prove optimality all possible solutions have to be enumerated (either explicitly or implicitly). Firstly, a simple branch-and-bound scheme will be elaborated. The difficulties encountered in this approach will serve as a link towards a branch-and-price approach.

\section{A branch-and-bound approach}

The most intuitive way of enumerating all solutions is to run through a triple loop. In the first loop all activities are enumerated. The second loop runs through all trainees that have to perform the activity under consideration. The third loop walks from the minimum number of consecutive periods till the maximum number of consecutive periods for the activity and trainee under consideration. Each time a non-available period is assigned, its cost is added to the total cost of the schedule. Before starting the enumeration the activities $k$ and the trainees within each activity $\left(j \in S_{k}\right)$ are sorted. Tests indicated that the best results are obtained when the activities are sorted in descending order of (average) difference between maximum and minimum number of consecutive periods of the trainees performing the activity. The trainees are sorted within each activity in descending order of occupation. By occupation we refer to the number of activities a trainee has to perform. In the pseudo-code below $\operatorname{cost}\left(a, j, t_{1}, t_{2}\right)$ represents the cost of scheduling trainee $j$ to perform 
activity $a$ from period $t_{1}$ until period $t_{2}$ (periods $t_{1}$ and $t_{2}$ are included) and $t c$ is the total cost of a (partial) schedule.

SORT();

DO RECURSION $(1,1)$;

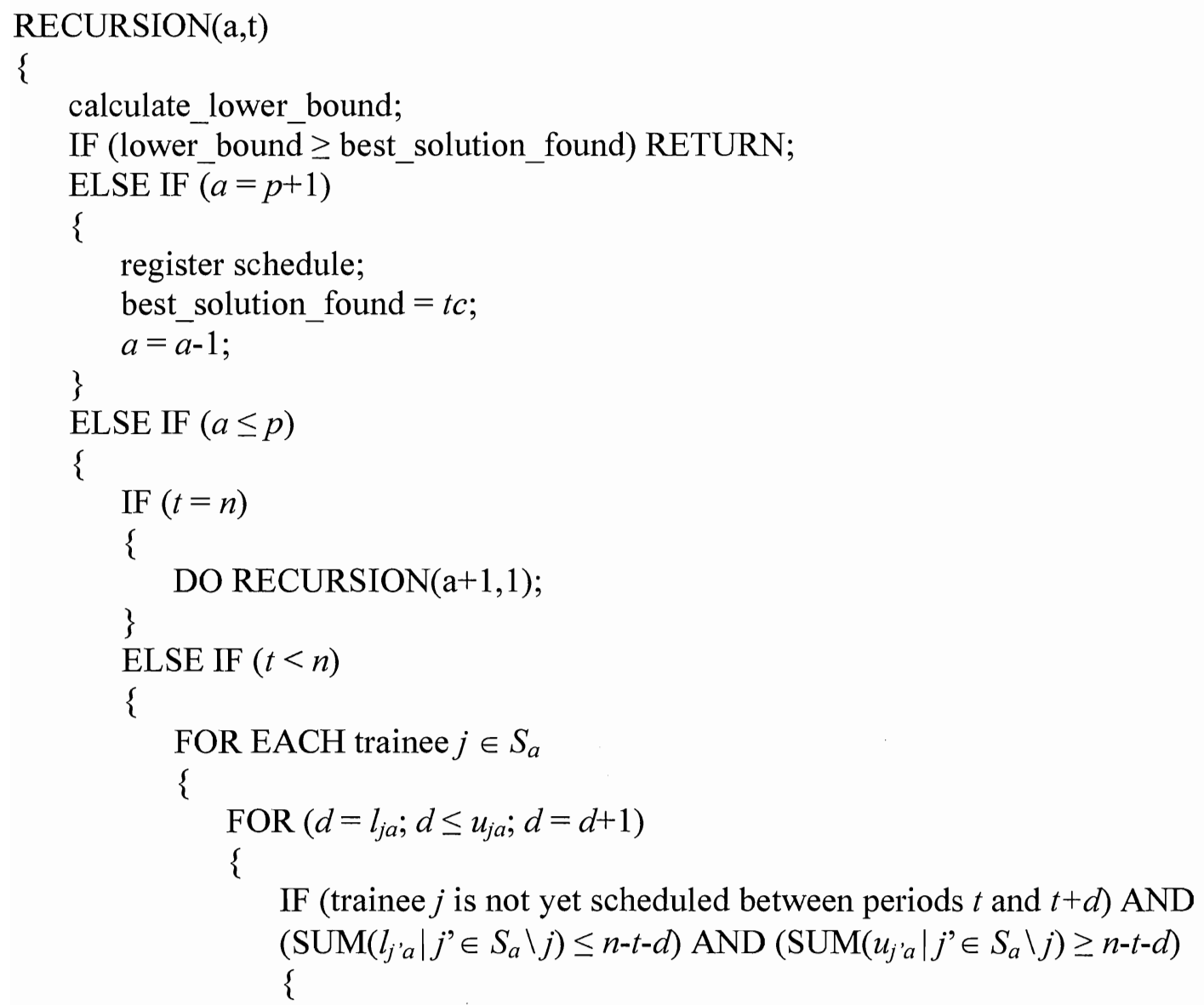

Schedule trainee $j$ to perform activity $a$ from period $t$ until period $t+d$;

$t c=t c+\operatorname{cost}(a, j, t, t+d)$

$S_{a}=S_{a} \backslash\{j\}$

DO RECURSION $(\mathrm{a}, t+d)$;

Undo scheduling of activity $a$ from period $t$ until period $t+d$;

$S_{a}=S_{a} \cup\{j\} ;$

$t c=t c-\operatorname{cost}\left(a_{2}, j, t, t+d\right)$;

\}

\} 
This algorithm explicitly enumerates all possible schedules. It should be clear that this number grows exponentially with the number of activities, the number of trainees per activity and the difference between the minimum and maximum number of consecutive periods required per activity per trainee. Obviously, the construction of many schedules could be interrupted at an early stage if one could prove that completing the current (partial) schedule never leads to a better schedule than the best schedule already found. To that purpose one should be able to calculate a strong (high) lower bound for the total cost of each (still to complete) partial schedule. The construction of partial schedules could also be terminated because of symmetry with an earlier generated schedule.

This branch-and-bound enumerating scheme has two disadvantages. The main problem is the calculation of strong lower bounds. Our lower bound calculation works as follows. For each period a minimum cost assignment problem is generated, in which the remaining activities are assigned to the available trainees. Each activity-trainee assignment cost equals the minimum cost to schedule the activity such that it 'covers' the time period for the considered trainee. A time period is covered by an activity for a certain trainee if the trainee is scheduled to perform the activity in the given time period. To find the minimum cost for each activity-trainee assignment, only the activity schedule at the minimum number of consecutive periods has to be considered, since longer activity schedules can only increase this cost. The construction of such a minimum cost assignment problem for period 1 is represented in figure 3.

\begin{tabular}{|c|c|c|c|}
\hline $\operatorname{tr} 1$ & $\operatorname{tr} 2$ & $\operatorname{tr} 3$ & $\operatorname{tr} 4$ \\
\hline & act 1 & & \\
\hline 4 & act 1 & & \\
\hline & act 1 & 4 & \\
\hline & 1 & act 1 & \\
\hline & & act 1 & \\
\hline & & act 1 & 2 \\
\hline 1 & & & act 1 \\
\hline & 4 & & act 1 \\
\hline act 1 & & 1 & \\
\hline act 1 & & & 3 \\
\hline
\end{tabular}

\begin{tabular}{l|cccc}
\multicolumn{5}{l}{ min. nr. consecutive periods: } \\
& $\operatorname{tr} 1$ & $\operatorname{tr} 2$ & $\operatorname{tr} 3$ & $\operatorname{tr} 4$ \\
\hline act 1 & 2 & 2 & 2 & 2 \\
act 2 & 2 & 2 & 2 & 2 \\
act 3 & 2 & 2 & 2 & 2
\end{tabular}

max. nr. consecutive periods:

\begin{tabular}{l|cccc} 
& $\operatorname{tr} 1$ & $\operatorname{tr} 2$ & $\operatorname{tr} 3$ & $\operatorname{tr} 4$ \\
\hline act 1 & 3 & 3 & 3 & 3 \\
act 2 & 3 & 3 & 3 & 3 \\
act 3 & 3 & 3 & 3 & 3
\end{tabular}

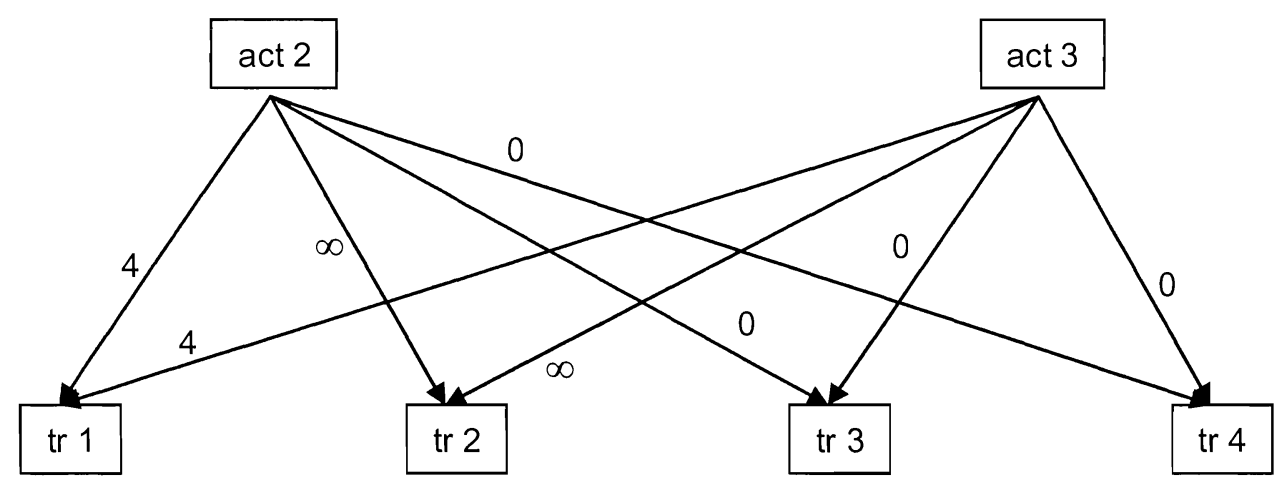

Figure 3: Assignment problem for period 1 in the partial schedule for example 1 represented above 
In this figure the numbers next to the arcs represent the assignment costs. For instance, to cover period 1 for trainee 1 with activity 2, a cost of 4 has to be incurred. All the trainee 2 assignment costs are $\infty$, since it is impossible to schedule an activity to cover period 1 for trainee 2 . Such an assignment problem can be solved very efficiently with a branch-andbound algorithm. For each activity, the available trainees are sorted in increasing order of assignment costs. Then, the activities are subsequently considered and the first still available trainee is assigned. After each trainee assignment, the trainee has to be excluded from being assigned to other activities. A lower bound for the assignment problem can be calculated by summing up all costs of the first still available trainee for each activity. The optimal solution value for the assignment problem in figure 3 is of course 0 . This is also the case for all assignment problems until period 7. The optimal costs for the assignment problems in period 8,9 and 10 are equal to 1 . If, however, for a certain time period an increase in the lower bound is obtained, then the algorithm cannot simply continue with solving the assignment problem of the next period, because the scheduling of an activity to cover the current period could also cover some later periods. Since a lower bound is searched, a best-case scenario has to be assumed. This means that the highest possible number of next periods have to be omitted. This is the maximum number of consecutive periods over all still to schedule activity-trainee combinations. In the example above, the lower bound can thus only be increased with 1, obtained from solving the assignment problem in period 8. Summarizing, given a partial schedule, a lower bound is found by solving at most $n$ assignment problems. The bound relaxation lies in two facts. Firstly, it is assumed that each activity can be scheduled for each trainee during its minimum number of consecutive periods, which obviously cannot be the case for all trainees in a feasible solution. Secondly, if during a certain time period a cost is inevitable, then it is assumed that the cost is incurred by scheduling an activity, such that a maximum number of next periods is also covered by this activity. The second relaxation could be tightened by doing the lower bound calculation a second time, but now starting at the end of the scheduling horizon and working backwards. A second way to tighten the second relaxation is to assume that each trainee has to perform each activity for only one period. In this way, assignment problems for next periods will never be omitted. This happens at the expense of a decrease in the assignment costs and thus an increase of the gap from the first relaxation. Obviously, the highest of the three bounds provides the best lower bound. This lower bound calculation is fast, but does not provide good lower bounds. Moreover, the quality of the bounds is highly dependent on the difference between the minimum and maximum number of consecutive periods (first relaxation) and the relative magnitude of the maximum number of consecutive periods (second relaxation).

A second problem with the branch-and-bound scheme is the way of traversing the search tree. The tree is searched in a depth-first way, which entails that, if accidentally a bad decision is made near the root of the tree, detection of the optimum could happen at a very late stadium in the search. A best-first search would solve this matter. However, since the number of active branches can be very large, this would happen at the cost of an important increase in memory usage.

Since both problems originate from the fact that optimality has to be proven, a heuristic search procedure seems to be the only alternative. A branch-and-price approach, however, provides an answer to both difficulties, while still maintaining the ability to prove the optimality of solutions. 


\section{A branch-and-price approach}

\subsection{An alternative formulation}

The integer program of [1.1]-[1.9] could be formulated in a totally different way. Observe that the problem can be seen as the scheduling of $p$ activity patterns. An activity pattern includes the scheduling of all trainees having to perform the activity. In figure 3 such a pattern is represented for activity 1 . For reasons that will become clear in a moment, an activity pattern will be called a column in the rest of this paper. Now, we can introduce new binary decision variables that explicitly incorporate these columns. Let binary decision variable $z_{k t}$ be defined as follows:

$$
\begin{aligned}
z_{k t} & =1, \text { if column } t \text { was chosen for activity } k \\
& =0, \text { otherwise. }
\end{aligned}
$$

Let $c_{k t}$ be the total cost of column $t$ for activity $k$ and $N C_{k}$ the total number of different columns for activity $k$. Let $a_{i j k t}$ equal 1 if trainee $j$ is scheduled during period $i$ in column $t$ for activity $k$. The model can then be formulated as follows:

$$
\mathrm{MIN} \sum_{k=1}^{p} \sum_{t=1}^{N C_{k}} c_{k t} z_{k t}
$$

S.T.

$$
\begin{array}{lll}
\sum_{k=1}^{p} \sum_{t=1}^{N C_{k}} a_{i j k t} z_{k t} \leq 1 & \forall i=1, . ., n \text { and } \forall j=1, . ., m & \lambda_{i j} \\
\sum_{t=1}^{N C_{k}} z_{k t}=1 & \forall k=1, . ., p & \gamma_{k} \\
z_{k t} \in\{0,1\} & \forall k=1, . ., p \text { and } \forall t=1, . ., N C_{k}
\end{array}
$$

The objective function is again the minimization of costs, but now expressed in terms of the new $z_{k t}$ variables. Constraint [1.11] states that each trainee can perform no more than one activity at the same time. Constraint [1.12] implies that exactly one column has to be selected for each activity. Remark that $a_{i j k t}$ is merely a coefficient in the constraint matrix of the new model, whereas $x_{i j k}$ was a decision variable in the old model. Tests revealed that the LP relaxation of this formulation provides a much stronger lower bound than that from the original formulation of [1.1]-[1.9]. The main drawback, however, is that this new model can have far more variables than can be reasonably attacked directly. Indeed, the number of columns increases dramatically with growing problem dimensions. It is however not necessary to enumerate all possible columns to solve the LP to optimality. The LP can be solved by using only a subset of the columns and can generate more columns as needed. This way of LP optimizing is called column generation. We iteratively add new columns and solve the restricted model until no more columns price out, i.e. no more columns with negative reduced cost can be found. Let $\lambda_{i j}$ represent the dual prices of 
restrictions [1.11] and let $\gamma_{k}$ represent the dual prices of restrictions [1.12]. The reduced cost of a new column $t$ for activity $k$ is given by:

$$
\begin{aligned}
& \gamma_{\mathrm{k}}+\sum_{i=1}^{n} \sum_{j=1}^{m}\left(p_{i j}+\lambda_{i j}\right) a_{i j k t} \\
& =\gamma_{\mathrm{k}}+c_{k t}+\sum_{i=1}^{n} \sum_{j=1}^{m} \lambda_{i j} a_{i j k t}
\end{aligned}
$$

In this expression $\gamma_{k}$ is non-positive and can be seen as the 'discount' for introducing a new column for activity $k$. This reward has to outperform the 'price' of the new column which is given by the remaining part in [1.14]. This price consists of two non-negative parts: the real price $c_{k t}$ and the price charged for 'consuming' timetable cells $(i, j)$ expressed by the dual prices $\lambda_{i j}$. Consequently, the LP is solved to optimality when no more columns can be found with negative reduced cost. The search is started by solving the master problem, stated in [1.10]-[1.12] $]^{1}$ for a limited number of columns. These initial columns are originated from a heuristic solution procedure. The master returns an objective value (which is an upper bound for the LP solution) and dual prices $\lambda_{i j}$ and $\gamma_{k}$. $\lambda_{i j}$ serves as a direct input for the objective function of the pricing problem (stated below), whereas $\gamma_{k}$ is needed to check the negativity of the reduced cost of a newly found column for activity $k$. Then, to check the optimality of the LP solution, a subproblem, called the pricing problem, is solved for each activity $k$. Let $b_{i j k t}$ equal 1 if in column $t$ activity $k$ starts during period $i$ for trainee $j$. The $t^{\text {th }}$ pricing problem for activity $k$ is given by:

$$
\begin{array}{ll}
\operatorname{MIN} \sum_{i=1}^{n} \sum_{j=1}^{m}\left(p_{i j}+\lambda_{i j}\right) a_{i j k t} & \\
\sum_{i=1}^{n} a_{i j k t} \geq l_{j k} & \forall j=1, . ., m \\
\sum_{i=1}^{n} a_{i j k t} \leq u_{j k} & \forall j=1, . ., m \\
b_{1 j k t}=a_{1 j k t} & \forall j=1, . ., m \\
b_{i j k t} \geq a_{i j k t}-a_{(i-1) j k t} & \forall i=2, . ., n \text { and } \forall j=1, . ., m \\
\sum_{i=1}^{n} b_{i j k t} \leq 1 & \forall j=1, . ., m \\
a_{i j k t}, b_{i j k t} \in\{0,1\} & \forall i=1, . ., m \text { and } \forall j=1, . ., m
\end{array}
$$

In this formulation the objective function [1.15] minimizes the reduced cost of the new column. Constraints [1.16] to [1.21] imply the restrictions each column has to satisfy.

\footnotetext{
${ }^{1}$ Remark that the integrality constraints are omitted
} 
Observe that in the pricing problem $a_{i j k t}$ is a decision variable instead of a coefficient. Columns with a negative reduced cost are added to the master problem and the master is again optimized. This process continues until no columns price out any more. As will be shown in a moment, the pricing problem can be solved very efficiently by means of a dynamic programming formulation.

When an integer problem is solved by an enumeration scheme in which the lower bound is calculated by a LP relaxation and the LP is solved through column generation, one speaks of a branch-and-price approach. One could ask why we didn't apply the original formulation [1.1]-[1.8] as a lower bound calculation in the enumeration scheme described above. This would avoid the need of column generation, but entails two important drawbacks. The first problem is that the number of branches (recursive calls) would still be large and thus the lower bound calculation, which is still computationally intensive, has to be done many times. The second and most important problem is the weakness of the lower bounds. This observation is completely in line with Barnhart et al. (1998) when they state that the LP relaxation frequently can be tightened by a reformulation that involves a huge number of variables.

In the next sections the branch-and-price algorithm will be elaborated. Firstly, an overview of the algorithm is given. Secondly, the pricing problem is discussed. Thirdly, four possible branching strategies are elaborated. Finally, some improvements to speed up the computation time will be studied and their importance will be discussed on the basis of test results.

\subsection{Branch-and-price algorithm overview}

In Figure 4 an overview of the branch-and-price algorithm is given. The algorithm starts with a heuristic search for an initial solution. This heuristic successively generates activity patterns (columns) without violating the no-overlap constraint. If the algorithm succeeds in finding a feasible solution, this solution is saved and an initial upper bound is registered. The number of trials is adjustable. The master is initialized with both the $p$ columns making up the best found solution and $p$ supercolumns (one per activity). Supercolumns have a very high objective coefficient $c_{t k}$ and all $a_{i j k t}$ equal to 0 . Consequently, supercolumns are not likely to enter the basis but are needed to ensure feasibility of the master at each stage of the branching scheme. Next, the algorithm enters the LP optimization loop. Every iteration of this loop consists of solving a number of pricing problems and adding columns to the master LP. When no more columns price out, the LP is optimized and the solution value is registered as the lower bound. If the solution is nonfractional, the solution is feasible and thus optimal. In the other case, branching has to be applied in order to find an integer solution. The algorithm again ends up in the LP optimization loop to determine a lower bound. Whenever this lower bound exceeds an already found upper bound, the loop is terminated and the search tree is backtracked. This process continues until an integer solution is found. The solution is saved and the solution value is registered as the new upper bound. The algorithm ends if the upper bound equals the lower bound or when backtracking leads back to the root node. 


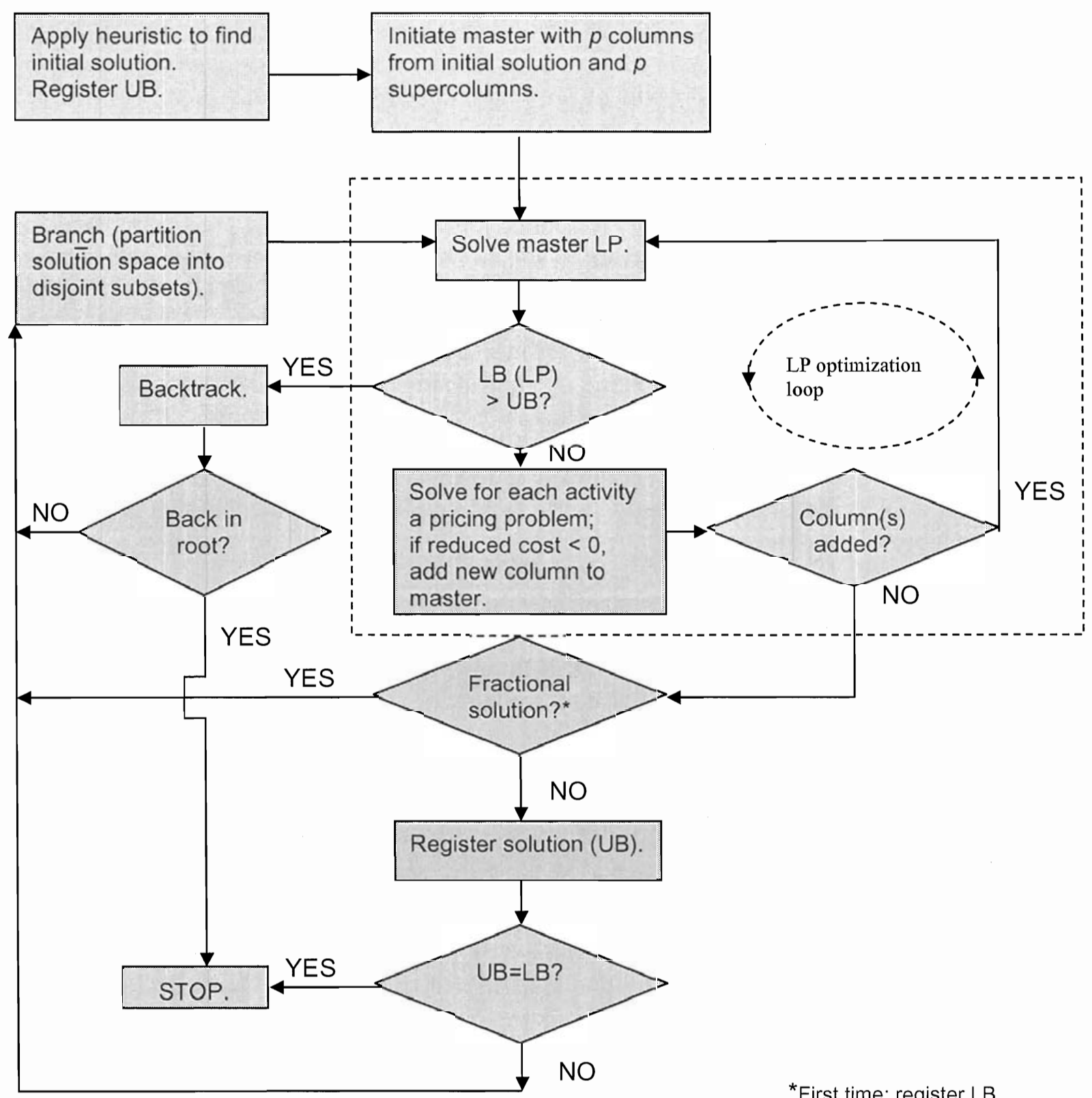

Figure 4: Branch-and-price algorithm overview

\begin{tabular}{|l|l|l|l|}
\hline 0 & 1 & 1 & 2 \\
\hline 0 & 1 & 1 & 1 \\
\hline 4 & 2 & 1 & 4 \\
\hline 2 & 1 & 0 & 0 \\
\hline 0 & 0 & 4 & 0 \\
\hline 1 & 5 & 3 & 1 \\
\hline
\end{tabular}

\begin{tabular}{|l|l|l|l|}
\hline 0 & 1 & 1 & 2 \\
\hline 0 & 1 & 1 & 1 \\
\hline 4 & 2 & 1 & 4 \\
\hline 2 & 1 & 0 & 0 \\
\hline 0 & 0 & 4 & 0 \\
\hline 1 & 5 & 3 & 1 \\
\hline
\end{tabular}

Figure 5: Pricing problem (left: optimal solution; right: $2^{\text {nd }}$ best solution) 


\subsection{The pricing problem}

The pricing problem can be described as a restricted shortest path problem. Given is a matrix of costs. This matrix has to be traversed from top to bottom in the cheapest possible way, while visiting each column exactly once between a minimum and maximum number of rows. Figure 5 represents the best and one of the several alternative second best solutions for an instance of a pricing problem for an activity of four trainees that all have to be scheduled between one and two periods in a time horizon of six periods. The pricing problem is solved with a forward dynamic programming approach. Dynamic programming (Bellman (1957); Dreyfus and Law (1977)) is a decomposition technique that first decomposes the problem into a nested family of subproblems. A possible way to divide the pricing problem in a set of nested subproblems is as follows. Let $T$ denote a set of trainees and $t_{l} \in T$ a trainee that is scheduled as the last one. The subproblem can be described as finding the cheapest way to reach a period $i \leq n$ with all trainees in $T$ scheduled and trainee $t_{l}$ scheduled as the last one. Let $\operatorname{cost}\left(i|T| t_{l}\right)$ represent the cost of the solution to this problem. If $c_{i, j}$ is the cost to assign period $i$ to trainee $j$ and we search a column for activity $k$, then $\operatorname{cost}\left(i|T| t_{l}\right)$ can be formulated recursively as follows:

$$
\operatorname{cost}\left(i|T| t_{l}\right)=\operatorname{MNN}_{l_{t_{l}} \leq d \leq u_{t_{l}, k}}\left\{\operatorname{MiN}_{t_{j} \in T \backslash\left\{t_{1}\right\}}\left\{\operatorname{cost}\left(i-d|T|\left\{t_{l}\right\} \mid t_{j}\right)+\sum_{b=1}^{d} c_{(t-d+b), t_{l}}\right\}\right\}
$$

The different values for $\operatorname{cost}\left(i|T| t_{l}\right)$ can be calculated working forward from the beginning until period $n$. All values are initialized with $+\infty$ except for each trainee $t_{j} \in S_{k}$ and for each $i=l_{t_{j} k} . . u_{t_{j} k}$ :

$$
\operatorname{cost}\left(i \mid\left\{t_{j}\right\} t_{\mathrm{j}}\right)=\sum_{b=1}^{i} c_{b, t_{j}}
$$

Once all calculations are done, the cheapest way to reach period $n$ can be found easily. Firstly, one searches the trainee that is to be scheduled last by solving the following expression:

$$
\operatorname{MDN}_{t_{t} \in S_{k}}\left\{\operatorname{cost}\left(n\left|S_{k}\right| t_{l}\right)\right\}
$$

Assume trainee $t_{m}$ is found with a total column cost of $\mathrm{c}^{*}=\operatorname{cost}\left(n\left|S_{k}\right| t_{m}\right)$. Then, the schedule is constructed backward step-by-step by searching for which trainee assignment the following expression holds:

$$
\mathrm{c}^{*}=\operatorname{cost}\left(n-d\left|S_{k}\right|\left\{t_{m}\right\} \mid t_{j}\right)+\sum_{\mathrm{b}=1}^{\mathrm{d}} c_{(n-b), t_{m}}
$$

This expression is checked for each trainee $t_{j} \in S_{k} \backslash\left\{t_{m}\right\}$ and for each $d=l_{t_{j} k} . u_{t_{j} k}$. If a match is encountered, the trainee is scheduled and both the current period and the set of already scheduled trainees are updated. This process continues until the last trainee is 
scheduled at the beginning of the scheduling horizon. Note that the major part of the required computation time goes to the calculations of all cost values. Once the cost values obtained, the schedule can be constructed in a relatively limited number of steps. The number of cost calculations obviously grows exponentially with the number of trainees that have to be scheduled. For realistic data (number of trainees) this number is not too large which results in acceptable solution times $(<1 \mathrm{~s})$. We tried to extend the dynamic program with a lower bound calculation giving rise to an $A^{*}$ algorithm. Preliminary results, however, indicated that the time spent in the lower bound calculation exceeds the time gained from node pruning. If larger networks (i.e. activities with more than ten trainees) are considered, a lower bound calculation is however indispensable.

An important advantage of the pure dynamic programming approach is that it can be easily extended to find the $b^{\text {th }}$ best solution instead of only the optimal solution. This property is very useful in a branch-and-price environment with branching on the column variables $z_{k t}$ (see 3.5.1). Our algorithm to find the $b^{\text {th }}$ best column reflects the same idea as the algorithm proposed by Jiménez and Marzal (1999) for computing the $K$ shortest paths in a network. To find the second best solution one first searches for the optimal solution as described above. Then, starting at the beginning of the time horizon all cost values being part of the found column are adapted to represent the second cheapest cost values. During this cost recalculation phase $\left|S_{k}\right|$ cost recalculations are made. For the example of figure 5 the recalculations are as follows. $\operatorname{cost}(2|\{1\}| 1)$ with initial value zero is now updated to $\infty$, since there is no other possibility to reach period 2 with trainee 1 scheduled and trainee 1 scheduled as the last one. $\operatorname{cost}(4|\{1,3\}| 3)$ with initial value one is now updated to $\infty$, since the only way to reach period 4 with trainees 1 and 3 scheduled and trainee 3 scheduled as the last one is to schedule trainee 1 during periods 1 and 2 and trainee 3 during periods 3 and 4 . The value of $\operatorname{cost}(5|\{1,2,3\}| 2)$ changes from one to two, since the cheapest way to reach period 5 with trainees 1,2 and 3 scheduled and 2 as the last one is now by scheduling trainee 1 during periods 1 and 2 , trainee 3 during period 3 and trainee 2 during periods 4 and 5. Finally, cost $(6|\{1,2,3,4\}| 4)$ is updated from two to three as the cheapest way to reach period 6 with all trainees scheduled and trainee 4 scheduled as the last one is now represented at the right of figure 5 . Note that the same cost can also be obtained when scheduling trainee 2 only during period 4 and trainee 4 during periods 5 and 6 . When the $\left|S_{k}\right|$ cost values are updated, the backward construction algorithm described above will now generate the second best column.

The old cost values (and the partial paths represented by these values), however, have to be saved in a list, because they could be part of the second best column and thus could be necessary during backward construction. This will be the case if the second best column has the same 'head' as the optimal column but a different 'tail', which is the case for the example in Figure 5. When during backward construction no trainee assignment can be found that matches [1.25], the list has to be scanned. In this case the list always contains a matching cost value. Note that once a cost value is retrieved in the list, the remaining trainee assignments can also be found in the list; they occupy the immediately preceding positions. Also during the cost recalculation phase, one should consider the list values as possible starting points when searching for the second best cost value to reach a certain node in the network. It should be clear that the extra computation time for finding the $b^{\text {th }}$ best solution only requires $2^{*} b^{*} \sum_{j \in S_{k}}\left(u_{j k}-l_{j k}\right)$ computation steps and $b^{*}\left|S_{k}\right|$ write 
instructions, which is negligible compared to the number of computation steps needed to calculate all initial cost values.

\subsection{Column addition}

An important characteristic of the branch-and-price algorithm is the number of columns that are added per master LP optimization. Three strategies can be distinguished. Firstly, we could add the most negative reduced cost column for each activity. Secondly, we could add only one column for all activities, i.e. the column with the overall most negative reduced cost. Finally, we could add the most negative reduced cost column for activity $k$, re-optimize the master and search for a new column for activity $k+1$. Remark that in this last strategy it is no longer possible to prune nodes based on Lagrange relaxation (see section 3.6.2), since the reduced costs of all activities, needed to calculate the lower bound, are no longer available. Obviously, for all three strategies, columns with nonnegative reduced cost are never added.

\subsection{Branching}

The LP relaxation of the master problem may not have an integral optimal solution. Branching refers to the process of partitioning the solution space to eliminate the current fractional solution. After branching it may be the case that there exists a column that would price out favorably, but is not present in the column pool. Applying standard branch-and-bound procedures to the master problem over the existing columns is unlikely to find an optimal, or good, or even feasible solution. To illustrate this point, a branch-andbound algorithm was written to find the best possible integral solution given the column pool after LP optimization. When the algorithm was run on the problem set, it never succeeded in finding a feasible solution, because the columns could not be combined into an integer solution.

Four binary branching schemes were implemented and extensively tested: branching on the column variables, branching on timetable cells, branching on immediate precedence relations and branching on normal precedence relations.

\subsubsection{Branching on column variables}

In this branching scheme branching happens by fixing the largest fractional variable $z_{k t}$ either to one (left branch) or to zero (right branch). It is however well known that 'direct' partitioning of the solution space, i.e. by fixing (or bounding) individual column variables, is not appropriate because of two reasons. Firstly, it could require significant alterations to the pricing problem and secondly, it yields an unbalanced branch-and-bound tree (Vanderbeck (2000)). The first problem is encountered along a branch, where a variable has been set to zero. Recall that $z_{k t}$ represents a particular schedule for activity $k$. Thus $z_{k t}=$ 0 means that this schedule is excluded. However, it is possible (and quite likely) that the next time the pricing problem is solved for the $k^{\text {th }}$ activity the optimal solution is precisely the one represented by $z_{k t}$. In that case it would be necessary to find the second best solution. At depth $l$ in the branch-and-bound tree we may need to find the $l^{\text {th }}$ best solution. We already showed that the dynamic programming approach for the pricing problem (see 
section 3.3) can be easily extended to handle this need and this at a negligible computational effort. The unbalanced branch-and-bound tree remains a problem, but also involves an advantage as faster detection of (sub)optimal integral solutions may be expected.

\subsubsection{Branching on timetable cells}

Since timetable cells are represented by the original variables, this branching scheme will also be referred to as branching on the original variables. When columns can be associated with paths in a network, a possible branching scheme consists of fixing single components of the arc incidence vector (Vanderbeck (2000)). If this branching principle is applied to our problem, it results in branching on the original $x_{i j k}$ variables. The next $x_{i j k}$ to branch on is found by selecting the largest fractional column $k^{\prime}$ and searching for this column the first timetable cell $(i, j)$ for which there exists another fractional column that includes the same time table cell $(i, j) . x_{i j k}$, is set to one in the left branch and to zero in the right branch. Alternatively, this branching rule can be seen as Ryan-Foster branching. Ryan-Foster branching, proposed by Ryan and Foster (1981) for set partitioning problems, identifies two rows, say $r$ and $s$, covered by different fractional columns. In the left branch rows $r$ and $s$ have to be covered by the same column and in the right branch by different columns. Observe that in our application row $r$ corresponds to one of the capacity constraints [1.11] and $s$ corresponds to one of the convexity constraints [1.12]. The main advantage of this branching scheme is that it does not destroy the structure of the pricing problem, because the resulting modifications simply entail amending the cost of the corresponding arc in the underlying network. If $x_{i j k}$ is set to zero, $c_{i, j}$ is set to $+\infty$ in the pricing problem of activity $k$. Else if $x_{i j k}$ is set to one, $c_{i, j}$ is set to $+\infty$ for all trainees $j \in S_{k}$ with $j \neq j$ '. A second advantage is the fact that this branching scheme yields a balanced branch-and-bound tree. The main drawbacks of this branching scheme are the large number of arcs $\left(x_{i j k}\right.$ 's) to choose from and the fact that a branching constraint that involves a single arc might not be very restrictive.

\subsubsection{Branching on immediate precedence relations}

In this branching scheme branching happens on immediate precedence relations within the trainees performing an activity. An immediate precedence relation for an activity $k$ between two trainees, say $j$ and $j$, implies that trainee $j$ has to perform activity $k$ either immediately before trainee $j$ '. In the twin node trainee $j$ cannot be scheduled immediately before trainee $j$ '. Upon detection of a fractional solution, the algorithm searches for two fractional columns for the same activity with different orderings in trainee assignments over the scheduling horizon. Then, an immediate precedence relation, which is satisfied by only one of both fractional columns, is implied. The main drawback of this branching scheme is that it is not guaranteed that it drives the solution completely to integrality. In other words, this branching scheme is not complete. Theoretically, it is possible that an optimal fractional solution is found in which all fractional columns for each activity have the same trainee ordering. If this would be the case, the algorithm rounds all fractional values to 1 and verifies whether or not the resulting solution contains an overlap. In the case of an overlap, only a lower bound instead of a feasible solution could be provided. 
Preliminary tests, however, indicated that this scenario occurs rarely. Similar to the case in which branching occurs on the timetable cells, application of this branching scheme preserves the structure of the pricing problem. An immediate precedence relation can be implied easily by simply amending the costs of certain arcs in the dynamic programming network. It is not immediately clear whether or not this branching scheme leads to more balanced branch-and-bound trees than the trees yielded when branching on the timetable cells. However, this branching scheme certainly leads to more balanced branch-and-bound trees than in the case of branching on the column variables.

\subsubsection{Branching on normal precedence relations}

This branching scheme is equal to the previous branching scheme, except that branching now occurs on normal precedence relations instead of immediate precedence relations. A normal precedence relation for an activity $k$ between two trainees, say $j$ and $j$, simply states that trainee $j$ has to perform activity $k$ either before or after trainee $j$ '. Obviously, this branching scheme also preserves the pricing problem structure and is also incomplete. Compared to the other three branching schemes, this branching scheme clearly yields the most balanced branch-and-bound trees. The restrictions implied in the left branches are similar to the ones implied in the right branches, i.e. if trainee $j$ cannot perform activity $k$ before trainee $j$ ', it means that trainee $j$ ' has to perform activity $k$ before trainee $j{ }^{2}$

\subsection{Speed-up techniques}

Once the column generator and branching scheme(s) are developed, we obtain a working branch-and-price algorithm. The performance of the algorithm is, however, strongly dependent on a number of speed-up techniques. The most important amongst these are discussed below.

\subsubsection{Initial heuristic}

The column pool is initialized with the columns making up an initial solution. The heuristic works as follows. Firstly, the activities $k$ are sorted such that the most constrained activities are considered first. Then, the first activity is scheduled optimally using the dynamic program of the pricing algorithm. All occupied timetable cells receive large costs $(+1000)$ in order to exclude overlaps when scheduling the next activities. In addition, the timetable cell costs just before and after the already scheduled activity are slightly decreased (-0.05), making them more attractive for scheduling the following activities. This prevents as much as possible the appearance of "holes", i.e. blocks of timetable cells that are too small to fit an activity in. Then, the next activity is considered taking into account the new timetable costs. This process continues until either all activities are scheduled or an activity cannot be scheduled any more due to an overlap. If all activities are scheduled, the total cost is compared with an earlier found solution and if lower, the upper bound is decreased. The changes to the timetable cell costs are made undone and the process restarts with the first activity. In order to avoid generation of the same columns as

\footnotetext{
${ }^{2}$ Observe that this is not the case when branching occurs on timetable cells or on immediate precedence relations.
} 
much as possible, the costs of the occupied timetable cells in the previous iteration are increased slightly $(+0.01)$ before starting a new iteration. The heuristic ends if it fails to improve the current best solution in a predetermined number of iterations.

\subsubsection{Lower bound calculation}

Our column generation scheme exhibits the tailing-off effect, i.e. requiring a large number of iterations to prove LP optimality. Instead of solving the linear program to optimality, i.e. generating columns as long as profitable columns exist, we could end the column generation phase based on bound comparisons. It is well known that Lagrangian relaxation can complement column generation in that it can be used in every iteration of the column generation scheme to compute a lower bound to the original problem with little additional computational effort (see e.g. Van den Akker and Hoogeveen (2000); Vanderbeck and Wolsey (1996)). If this lower bound exceeds an already found upper bound, the column generation phase can end without any risk of missing the optimum. Using the information from solving the reduced master and the information provided by solving a pricing problem for each activity $k$, it can be shown (see e.g. Hans, 2001) that a lower bound is given by:

$$
\delta+\sum_{k=1}^{p} R C_{k} \theta_{k}
$$

where $\delta$ is the objective value of the reduced master, $R C_{k}$ is the reduced cost of a newly found column for activity $k$ and $\theta_{k}$ is a binary variable equal to 1 when $R C_{k}$ is non-negative and set to zero, otherwise. This lower bound is referred to as the Lagrangian lower bound, since it can be shown that it equals the bound obtained by Lagrange relaxation. In addition with an upper bound it can also be used to fix variables. When the reduced cost of a variable $z_{k t}$ is larger than $U B-L B$, we know from linear programming theory that $z_{k t}=0$ in any solution with a value less than $U B$. Hence, that variable can be fixed in the current node and in all nodes below that node. Analogously, when the reduced cost is smaller than $L B-U B$ then $z_{k t}=1$ in any solution with a value less than $U B$.

\subsubsection{Initial network restriction}

Recall that, to price out a new column, a shortest path network problem is solved by applying a forward dynamic program approach. For problems in which these networks are very large, the pricing problems are the bottleneck of the algorithm. We distinguish two ways of decreasing the required solution times of the pricing problems. Firstly, one could initially restrict these networks. Concretely, arcs with positive non-availability costs are excluded during the early phase of each LP optimization loop. When no more columns can be found with negative reduced cost, these arcs are restored. The benefits are two-fold. Firstly, the required time of the pricing algorithm dramatically decreases during the early phase of column generation. Secondly, from the start on, the algorithm is forced to price out qualitatively good columns. 


\subsubsection{Dynamic programming with upper bound pruning}

A second way of reducing the computational effort to price out a new column was already mentioned in section 3.3 and involves an extension of the forward dynamic recursion with bound pruning. Whenever the forward dynamic program reaches a leaf node in the network, the cost value of the path can be compared with the best found solution and, if lower, registered as the new upper bound. This enables us to stop the construction of partial paths as soon as the associated cost value exceeds the upper bound. Remark that the cost value of a partial network cannot be decreased when extending the path, since all nonavailability costs and dual prices of the non-overlap constraints are non-negative. As also mentioned in section 3.3, the partial cost value could be increased with a lower bound calculation for scheduling the remaining trainees. Preliminary tests, however, showed that this didn't turn out to be beneficial. Finally, remark that bound pruning is not possible if it could be required to find the $2^{\text {nd }}, 3^{\text {rd }}, \ldots, k$ th best path, since these could be pruned. Hence, when branching takes place on the column variables, a pure dynamic program is applied to price out new columns.

\subsubsection{Master LP optimization}

An important computational issue relates to the optimization of the master linear program. When new columns are added and the master is re-optimized, the (dual) simplex algorithm could be started either from an empty base or from the optimal base of the previous iteration. Tests revealed that the LP is optimized fastest when started from an advanced base.

\subsubsection{Cost varying horizon}

To limit the solution space as much as possible, we implemented the idea of a cost varying horizon. This idea is equivalent with a time varying horizon in exact algorithms for the Resource Constrained Project Scheduling Problem (Demeulemeester and Herroelen, 1992).

When implementing a cost varying horizon, one could distinguish between a maximum and minimum bounding search strategy. Both strategies are different with respect to the value of the upper bound. In minimum bounding search the upper bound reflects the best found solution. When it is important to prove the optimality of a solution, a maximum bounding approach can be more effective than a minimum one. In maximum bounding search the upper bound is set to the first integer equal to or higher than the LP lower bound. If the algorithm succeeds in finding a solution with a total cost equal to this upper bound, we found an optimal solution. Otherwise, both the upper and lower bound are increased with one, the column pool is re-initiated with the columns making up the LP optimum and the algorithm tries to find a solution equal to this new upper bound. This approach corresponds to best-first search in branch-and-bound, as the first solution obtained is also the optimal solution. Tests indicated that maximum bounding search slightly decreases computation times at the expense of not providing (sub)optimal solutions during search. 


\subsubsection{Column elimination}

The idea of column elimination is inherent in all branching schemes except for the column-based branching scheme. To fully exploit the column-based branching strategy, the branching scheme was extended in that it also inherits the idea of column elimination. The solution time of the master LP grows strongly with the number of columns in the master, even when their associate column variables $z_{k t}$ cannot be positive in a feasible solution. After branching, an important number of already generated columns could be excluded from the master. If a particular column, say $z_{k^{\prime} t}$, is set to one, all other columns $z_{k^{\prime} t}$ with $t \neq t^{\prime}$ are excluded implicitly because of the convexity constraint [1.12] in the master. To speed up the computation time of the master, these columns can be excluded explicitly from the master (by eliminating them). Similarly, all columns having an overlap with column $z_{k^{\prime} t}$ ' can be excluded as well, due to the non-overlap constraints [1.11]. Observe that eliminated columns have to be saved, since they have to be reentered upon backtracking. Obviously, if column $z_{k^{\prime} t^{\prime}}$ is set to zero, no columns but $z_{k^{\prime} t^{\prime}}$ can be left out. Column elimination is inherent when branching occurs on the original variables. Consider the situation in which $x_{i^{\prime} j^{\prime} k^{\prime}}$ is set to one. All columns $z_{k^{\prime} t}$ not including timetable cell $\left(i^{\prime}, j^{\prime}\right)$ (i.e. having $a_{i j^{\prime} k^{\prime} t}=0$ ) will be left out. Similarly, all columns $z_{k t}$ with $k \neq k$ ' including timetable cell $\left(i^{\prime}, j j^{\prime}\right)$ (i.e. having $a_{i{ }^{\prime} j^{\prime} k t}=1$ ) will be removed as well. If $x_{i^{\prime} j^{\prime} k}$ ' is set to zero, the reverse applies. Column elimination is also inherent in the immediate and normal precedence relation branching schemes. Columns that do not satisfy the introduced precedence relations will be eliminated explicitly out of the master.

The same reasoning leads to the artificial adaptation of dual prices when branching occurs on the column variables. During preliminary tests of the algorithm, columns were generated that share timetable cells with already branched-to-one columns. Obviously, these columns can never enter the basis. The algorithm was adapted in that the dual prices of all timetable cells making up branched-to-one columns are increased with an artificially high value. Observe again that these artificial cost adaptations are inherent in the case branching is done on timetable cells and on immediate or normal precedence relations.

\subsubsection{Subgradient optimization}

Instead of solving the master problem with a standard simplex algorithm, subgradient optimization applied on the Lagrangian relaxation of the master could be used to find the dual prices. Excellent expositions on how to exploit Lagrangian relaxation and subgradient optimization techniques in combination with column generation can be found in Peeters (2002) and Jans (2002). If we relax the capacity constraints [1.11], dualizing them into the objective function [1.10], we obtain the Lagrange problem of [1.10]-[1.13]:

$$
\begin{array}{rlrl}
\operatorname{MIN} \sum_{k=1}^{p} \sum_{t=1}^{N C_{k}}\left(c_{k t}+\sum_{i=1}^{n} \sum_{j=1}^{m} a_{i j k t} \lambda_{i j}\right) z_{k t}-\sum_{i=1}^{n} \sum_{j=1}^{m} \lambda_{i j} & \\
\sum_{t=1}^{N C_{k}} z_{k t}=1 & \forall k & =1, . ., p \\
z_{k t} \in\{0,1\} & \forall k & =1, . ., p \text { and } \forall t=1, . ., N C_{k}
\end{array}
$$


We have implemented a standard subgradient optimization scheme for setting the dual prices $\lambda_{i j}$. At step $r+1$, the dual prices are updated as follows:

$$
\begin{aligned}
& \lambda_{i j}^{r+1}=\max \left\{0, \lambda_{i j}^{r}-\sigma\left(1-\sum_{k=1}^{p} \sum_{t=1}^{N C_{k}} a_{i j k t} z_{k t}^{r}\right)\right\} \quad \forall i=1, . ., n \text { and } \forall j=1, . ., m \\
& \text { with } \sigma=\frac{\omega\left(U B-Z_{L A G}\left(\lambda_{i j}^{r}\right)\right)}{\sum_{i=1}^{n} \sum_{j=1}^{m}\left(1-\sum_{k=1}^{p} \sum_{t=1}^{N C_{k}} a_{i j k t} z_{k t}^{r}\right)}
\end{aligned}
$$

In this expression $\sigma$ can be seen as a stepsize. $Z_{L A G}\left(\lambda_{i j}^{r}\right)$ is the optimal objective value [1.27] of the Lagrange dual problem for a given set of dual prices $\lambda_{i j}^{r}$ at step $r$ and $z_{k t}^{r}$ indicates the corresponding optimal value of column $t$ for activity $k$. The optimal solution to the Lagrange dual problem [1.27]-[1.29] is always integral and easily found by setting for each activity the $z_{k t}$ with the lowest cost equal to 1 and all other $z_{k t}$ 's equal to 0 . $U B$ is the best known integer solution. $\omega$ is initially set to 1.5 and is halved each time the lower bound has failed to increase for a fixed number of iterations. After a predetermined number of iterations we stop the updating and we obtain the approximate dual prices $\lambda_{i j}$. In order to determine whether or not a new column prices out and thus should be added to the master, one has to calculate the reduced cost of the new column. The reduced cost could be calculated using the approximating dual prices given in [1.28] or the optimal dual prices obtained from the last solved master. If the approximating dual prices are used, one still needs to calculate the dual price $\gamma_{k}$ of the convexity constraint [1.12]. It can be shown that the dual price of the convexity constraint for activity $k$ is approximated by the cost of the optimal column, as defined in the first term of [1.27].

\section{Computational results}

\subsection{Test set}

In order to study the computational performance of the algorithm, a test set was generated. Firstly, the six factors that have an influence on the complexity of the problem were identified. These are the number of periods, the number of trainees, the number of activities, for each activity the number of trainees performing the activity, the difference between the maximum and minimum number of consecutive weeks further referred to as the range and finally the magnitude of the costs. Consider the following settings for these six factors: 
Table I: Design of experiment

\begin{tabular}{c|cccccc}
$\begin{array}{c}\text { Factor } \\
\text { setting }\end{array}$ & $\begin{array}{c}\text { Nr. } \\
\text { periods }\end{array}$ & $\begin{array}{c}\text { Nr. } \\
\text { trainees }\end{array}$ & $\begin{array}{c}\text { Nr. activities } \\
\text { (\% of nr. } \\
\text { trainees) }\end{array}$ & $\begin{array}{c}\text { Nr. trainees per } \\
\text { activity (\% of nr. } \\
\text { trainees) }\end{array}$ & Range & $\begin{array}{c}\text { Magnitude } \\
\text { of costs }\end{array}$ \\
\hline 1 & 18 & 6 & 60 & 60 & 1 & 1 \\
2 & 35 & 8 & 75 & 75 & 2 & $\cup(1,5)$ \\
3 & 52 & 10 & 90 & 90 & 3 & \\
4 & & 12 & & random(75) & 4 & \\
5 & & & & & random(2) & \\
6 & & & & & random(3) &
\end{tabular}

Observe that the number of activities and the number of trainees having to perform an activity is expressed as a percentage of the number of trainees. For instance, a test problem with 10 trainees and $90 \%$ activities includes 9 activities. Note also that the number of activities cannot exceed the number of trainees, because otherwise not all activities can be performed. The ratio $\mathrm{nr}$. activities over nr. trainees represents the total schedule occupation percentage. Recall that the remaining part of the schedule has to be filled up with activities for which the consecutiveness is not important. $\operatorname{random}(x)$ indicates that the factor setting is uniformly distributed with an average of $x$. For instance, the range setting random(2) means that the ranges are generated randomly in such a way that the average amounts to 2 . If the magnitude of the costs is 1 , it means that all non-available time periods, which are generated randomly for each trainee, have a cost of 1 . Alternatively, these cost values are drawn from a uniform distribution between 1 and 5 .

According to these factor settings, problem instances were generated with randomness on both the activity-trainee assignments and the non-available periods. In order to exclude non-feasible and trivial problems as much as possible, the trainee occupations were kept more or less at the same level. Without loss of generality all non-availability costs are assumed to be integral. The total number of periods containing positive costs equals 3,4 or 5 for problems with respectively 18,35 and 52 periods. If we generate three problem instances per factor setting, we obtain $3 *(3 * 4 * 3 * 4 * 6 * 2)=5184$ problem instances. In order to decrease this number, we decided to subsequently fix the first three factors and the next two factors (the fourth and fifth factor) at an intermediate level, making us end up with $3 *(4 * 6 * 2)+3 *(3 * 4 * 3 * 2)=360$ problem instances.

\subsection{Proven optimal solutions}

In order to find (proven) optimal solutions, all above discussed speed-up techniques turned out to be useful to reduce computation times, except, somewhat surprisingly, for subgradient optimization (see section 3.6.7). In this part we present optimal solutions and computation times of all problems instances. All our experiments were performed on a 2.4 $\mathrm{GHz}$ Pentium 4 PC with the Windows XP operating system. The algorithm was written in MS Visual C++.NET and linked with the CPLEX 8.1 optimization library. All speed-up techniques described above were incorporated in the algorithm, except for subgradient optimization (see Section 3.6.7), since it could not improve solution times. We apply maximum bounding search on all 360 problem instances and distinguish between the four branching strategies. All computation times are given in seconds. The results are 
represented in Appendix 1. The first column contains the name of the problem instance. All problems are named "DOEabcdef_g", which can be interpreted as follows:

- $\mathrm{a}$ is the factor setting for the number of periods,

- $b$ is the factor setting for the number of trainees,

- $\mathrm{c}$ is the factor setting for the number of activities,

- $\mathrm{d}$ is the factor setting for the number of trainees for each activity,

- $\mathrm{e}$ is the factor setting for the range,

- $\mathrm{f}$ is the factor setting for the magnitude of costs,

- $\mathrm{g}$ is a replication number.

The second column contains the optimal LP objective value at the root node (before branching), the heuristic solution, and the optimal integer solution value (after branching). The next columns contain respectively the required CPU time (in seconds), the number of generated columns and the number of nodes in the branch-and-bound tree for each branching scheme. The time limit for each problem was 600 seconds. If the optimum was not found (or proven) within this time limit, this is indicated with a " $>600 "$ ". Recall that, since a maximum bounding search is applied, no solution is found when the algorithm fails to find the optimum. If the precedence-related branching schemes could not branch until a non-fractional solution, this is indicated with "Fract" equal to 1.

\subsection{Discussion of results}

In this section, we summarize the most important findings from our computational experiments.

In table II the number of problems that could be solved to optimality within 10 minutes is given for each branching scheme together with the average computation times. The second row $(*)$ contains the average times for only those problems for which all four branching schemes succeeded in finding (and proving) the optimal solution within 600 seconds. In the third row $(* *)$ average times are calculated based on all problems. For these calculations, 600 seconds were assigned to those problems for which no optimal solution was found within 600 seconds.

Table II: Comparison of branching schemes

\begin{tabular}{|c|c|c|c|c|}
\hline Branch on: & $\begin{array}{c}\text { column } \\
\text { variables }\end{array}$ & timetable cells & $\begin{array}{c}\text { immediate prec. } \\
\text { relations }\end{array}$ & $\begin{array}{c}\text { normal prec. } \\
\text { relations }\end{array}$ \\
\hline $\begin{array}{c}\text { Nr. solved to } \\
\text { optimality }\end{array}$ & 311 & 319 & 281 & 281 \\
\hline $\begin{array}{c}\text { Average comp. } \\
\text { time* (s) }\end{array}$ & 14.5 & 19.0 & 22.9 & 42.1 \\
\hline $\begin{array}{c}\text { Average comp. } \\
\text { time** (s) }\end{array}$ & 112.0 & 114.1 & 161.1 & 171.5 \\
\hline
\end{tabular}


A first important observation is that the two precedence-related branching schemes are clearly outperformed by the first two branching schemes. A second observation is that, although branching on timetable cells yields more problems solved to optimality, the required computation times are generally higher than those for the column-based branching scheme. This is a clear indication of the appearance of unbalanced branch-andbound trees when branching occurs on the column variables.

In the following graphs the results are visualized per factor combination. The first four graphs (Figure 6) summarize the results as a function of varying number of trainees for each activity and range. The number of periods, trainees and activities are fixed at an intermediate level and the non-availability costs are always 1 . The columns represent average computation times over three test instances per factor setting for each branching scheme. The first column indicates branching on the column variables, the second branching on the timetable cells and the third and the fourth branching on immediate and normal precedence relations.
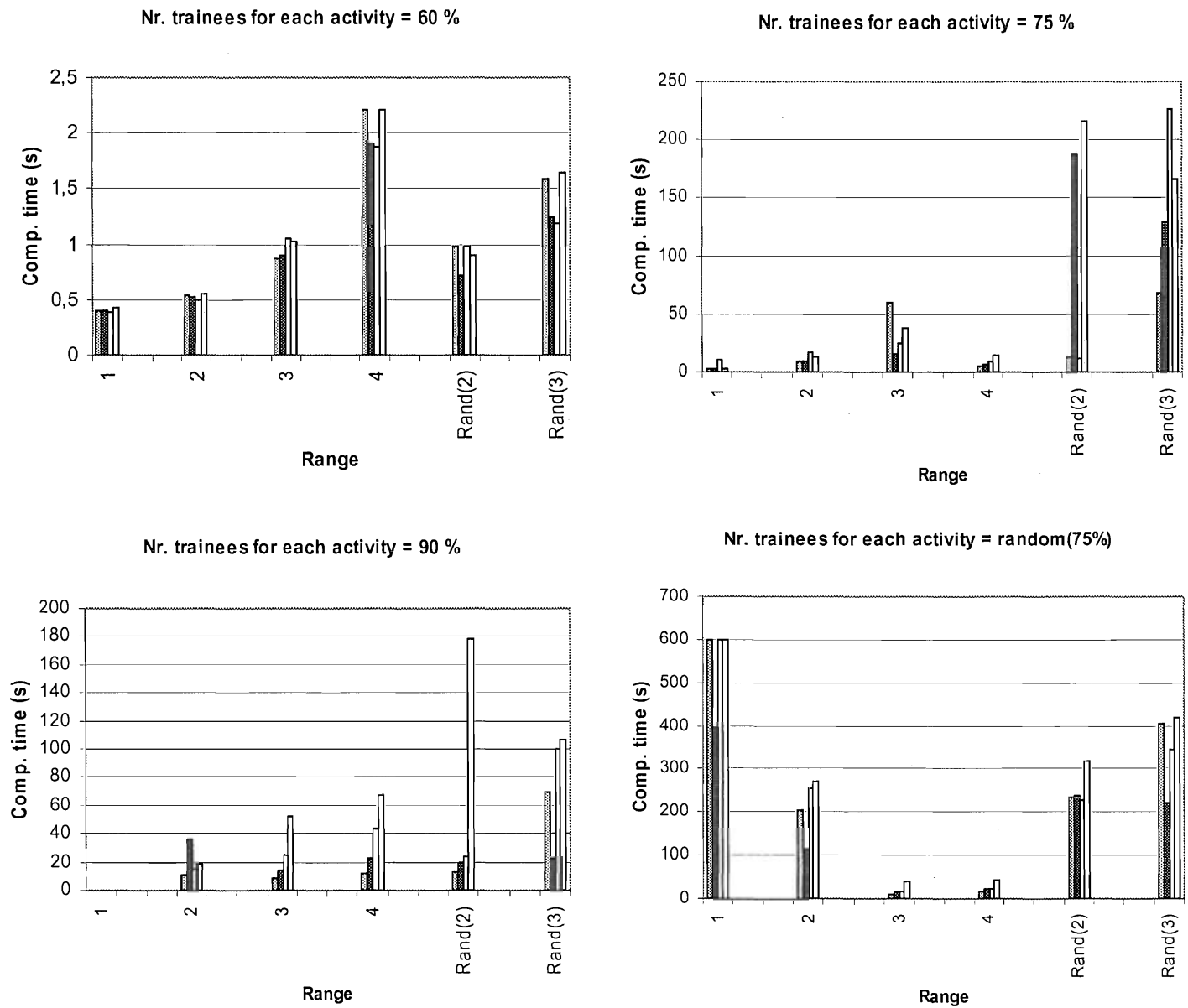

Figure 6: Comp. times for nr. periods $=35, \mathrm{nr}$. trainees $=8, \mathrm{nr}$. activities $=6$ and magnitude of costs $=$ 
The conclusions with respect to these graphs are three-fold. Firstly, the computation times grow exponentially with the number of trainees per activity. The results in graph 4 suggest that randomness on this factor complicates the problem only if the range is small. Secondly, the influence of the range on the complexity of the problem is far less important. The reason is that the number of possible columns for each activity grows exponentially with the number of trainees (all possible permutations) whereas this number grows only linearly with the range. All four graphs indicate that randomness on the range tends to complicate the problem. Thirdly, branching on the columns and timetable cells seem to outperform the two precedence-related branching schemes.

The next set of four graphs (Figure 7) show the same information with the only difference that the magnitude of the costs are now randomly distributed between 1 and 5 .

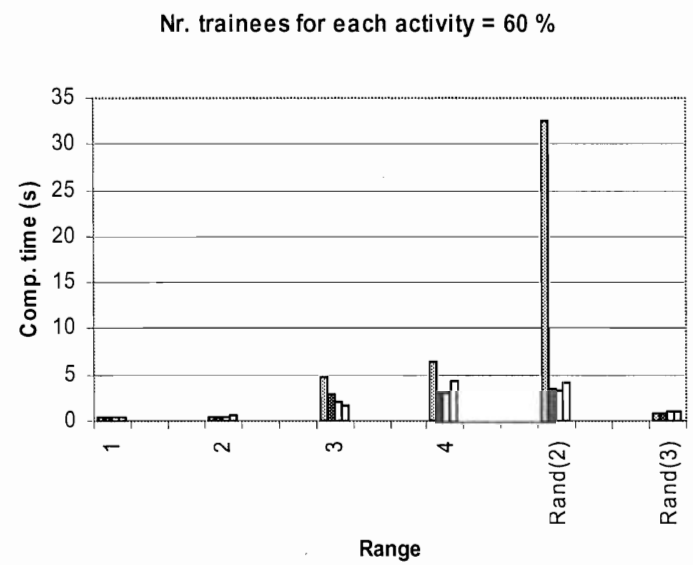

Nr. trainees for each activity $=90 \%$

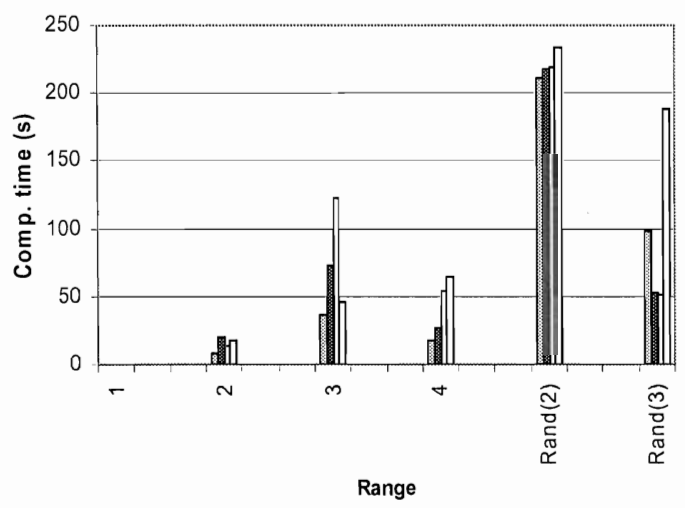

Nr. trainees for each activity $=75 \%$

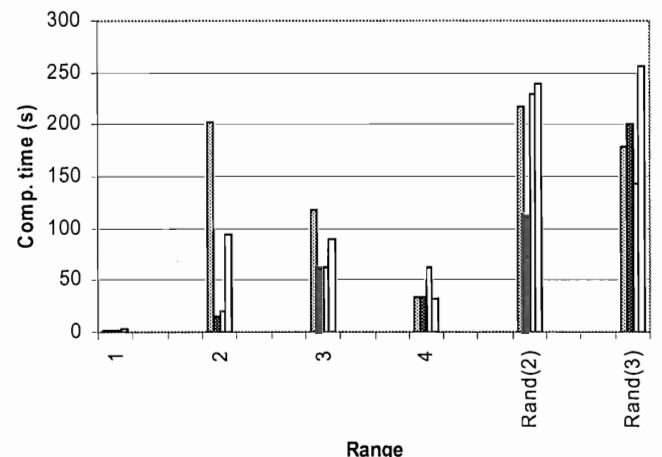

Nr. trainees for each activity $=\operatorname{rand}(75 \%)$

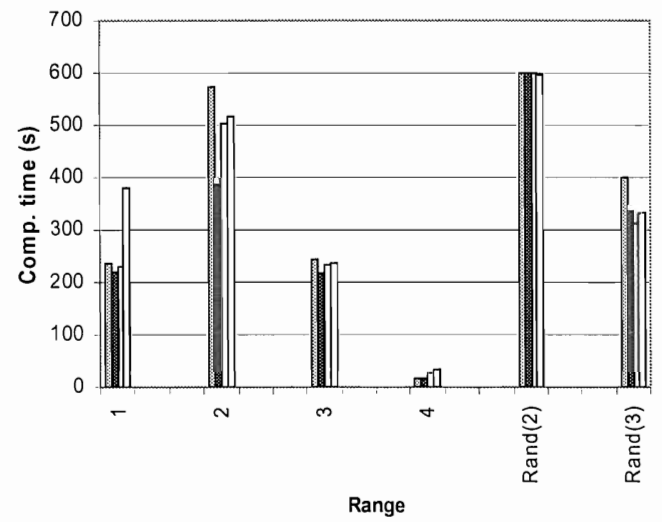

Figure 7: Results for nr. periods $=35, \mathrm{nr}$. trainees $=8, \mathrm{nr}$. activities $=6$ and magnitude of costs $=$ $\mathrm{U}(1, \mathbf{5})$

These graphs confirm all previous conclusions. With respect to the comparison of the first two branching schemes, one can observe that branching on the columns now performs 
significantly worse. This can be explained by the fact that the randomness on the magnitude of the costs makes the quality of the LP lower bound decrease, which of course brings more harm to the unbalanced column branching than to the balanced timetable cell branching.

The next three graphs show the results for varying number of periods, trainees and activities while the fourth and the fifth factor are fixed at an intermediate level. We distinguish again between the case in which the non-availability costs are always 1 and the case in which these costs are uniformly distributed between 1 and 5.
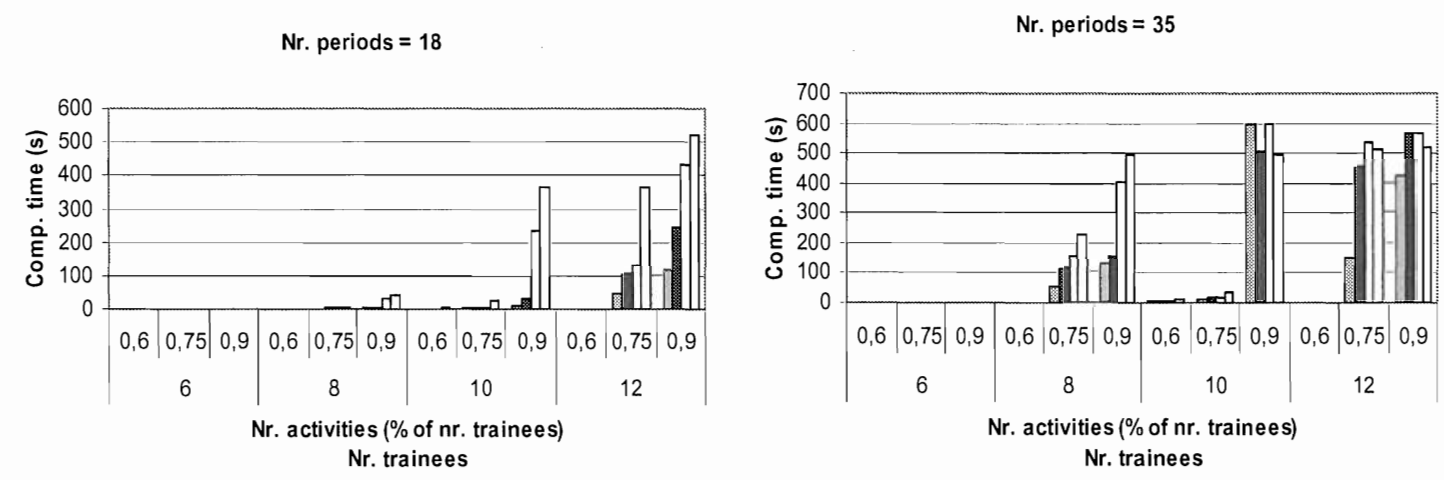

Nr. periods $=52$

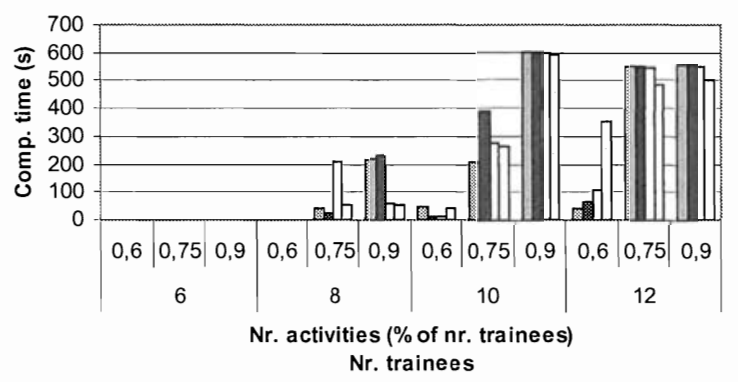

Figure 8: Results for $\mathrm{nr}$. trainees for each activity $=75 \%$, range $=3$ and magnitude of costs $=1$

Nr. periods $=18$

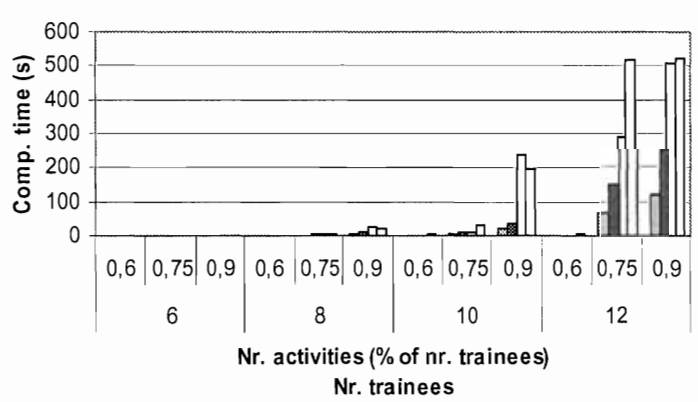

Nr. periods $=35$

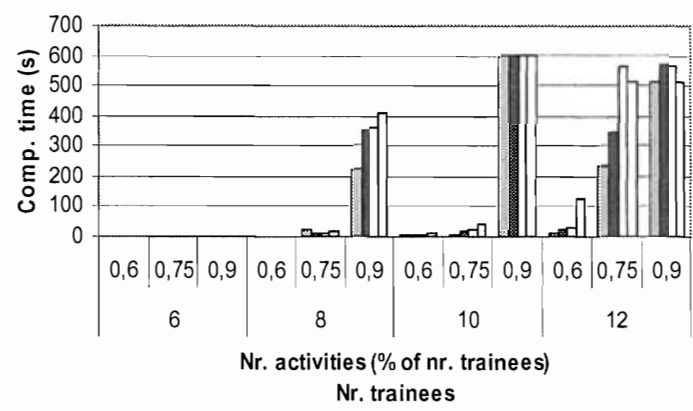




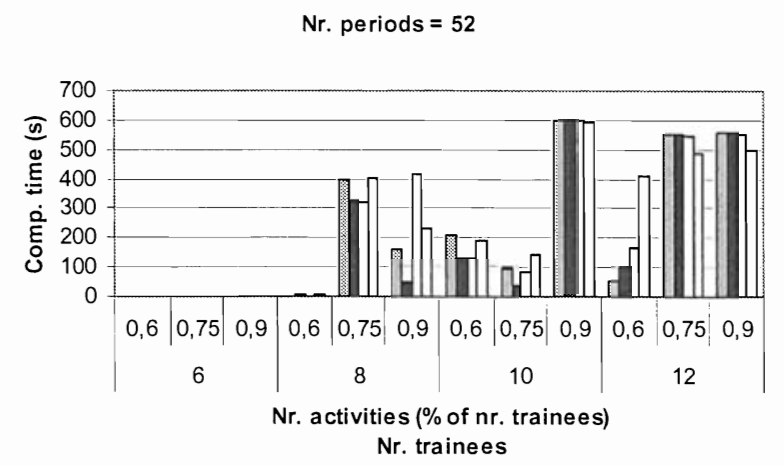

Figure 9: Results for $n r$. trainees for each activity $=75 \%$, range $=3$ and magnitude of costs $=U(1,5)$

From these graphs one may conclude that both the number of activities and the number of trainees have a significant impact on the complexity of the problem. With a few exceptions the algorithm was not able to solve problems with 12 trainees and 11 activities to optimality within the time limit of 600 seconds. Furthermore, the results indicate that the impact of the number of periods on the complexity of the problem is rather small. Similar to the previous results, the precedence-related branching schemes are outperformed by the first two branching schemes. Branching on the columns seems to perform best when the magnitude of the costs is always 1 , whereas when these cost values are uniformly distributed between 1 and 5, branching on the timetable cells seems to be the most robust way of branching.

\section{Contributions of speed-up techniques}

In order to gain some insights into the contributions of the different speed-up techniques, an experiment was performed including all 307 problems for which an optimal solution was found within 600 seconds for both the first and the second branching scheme. Besides all eight speed-up techniques (see section 3.6) the influence of the two alternative ways of column addition (see section 3.4) was investigated. The results are presented in Table 3 and visualized in Figure 10. The first row of Table 3 contains the average computation times for the basic algorithm, i.e. the branch-and-price algorithm including all speed-up techniques except for subgradient optimization (see section 4.2). Rows 2 to 9 contain the average computation times when the respective speed-up technique was omitted (or included in the case of subgradient optimization). Note that the effects are not cumulative, i.e. the algorithm always included all but one speed-up technique. Row 10 gives the computation times when only one column, i.e. the overall best (most negative reduced column), was added after each master optimization. Finally, row 11 contains the computation times when the master was re-optimized after the generation of only one column, instead of one column for each activity. Recall that the dynamic program could not be extended with upper bound pruning in the first branching scheme, since the $2^{\text {nd }}, 3^{\text {rd }}$, $\ldots, \mathrm{k}^{\text {th }}$ best column may be required. Recall also that column elimination is inherent in the second branching scheme. 
Conclusions with respect to this experiment are twofold. Firstly, the way of column addition plays a major role in fast convergence of column generation. Adding only one (optimal) column per master optimization seems to be outperformed by adding $k$ (suboptimal) columns per master optimization. The main reason for the large difference between (10) and (11) is probably the impossibility in (11) to prune nodes based on Lagrange relaxation.

Secondly, the results clearly indicate the positive impact of all speed-up techniques except for subgradient optimization. The initial heuristic and initial network restrictions turned out to have the smallest impact on the computation times.

Table III: Contributions of speed-up techniques

\begin{tabular}{lrrr} 
& \multicolumn{2}{c}{$\begin{array}{c}\text { Average computation time (s) } \\
\end{array}$} & $\begin{array}{c}\text { Branching } \\
\text { Branching on } \\
\text { on columns timetable cells }\end{array}$ \\
\hline Basic algorithm & $(1)$ & 36.00 & 36.17 \\
Without initial heuristic (section 3.6.1) & $(2)$ & 37.94 & 41.60 \\
Without initial network restriction (section 3.6.3) & $(3)$ & 36.14 & 42.60 \\
DP without upper bound pruning (section 3.6.4) & $(4)$ & - & 49.51 \\
Without Lagrange dual pruning (section 3.6.2) & $(5)$ & 42.57 & 49.96 \\
Minimum bounding search strategy (section 3.6.6) & $(6)$ & 56.66 & 60.97 \\
Solving master LP starting from empty basis (section 3.6.5) & $(7)$ & 55.92 & 67.80 \\
With subgradient optimization ${ }^{3}$ (section 3.6.8) & $(8)$ & 65.88 & 81.44 \\
Without column elimination first branching strategy (section 3.6.7) & $(9)$ & 93.46 & - \\
Search $k$ columns, add 1 column per master optim. (section 3.4) & $(10)$ & 72.33 & 76.03 \\
Search 1 column, add 1 column per master optim. (section 3.4) & $(11)$ & 141.09 & 148.31
\end{tabular}

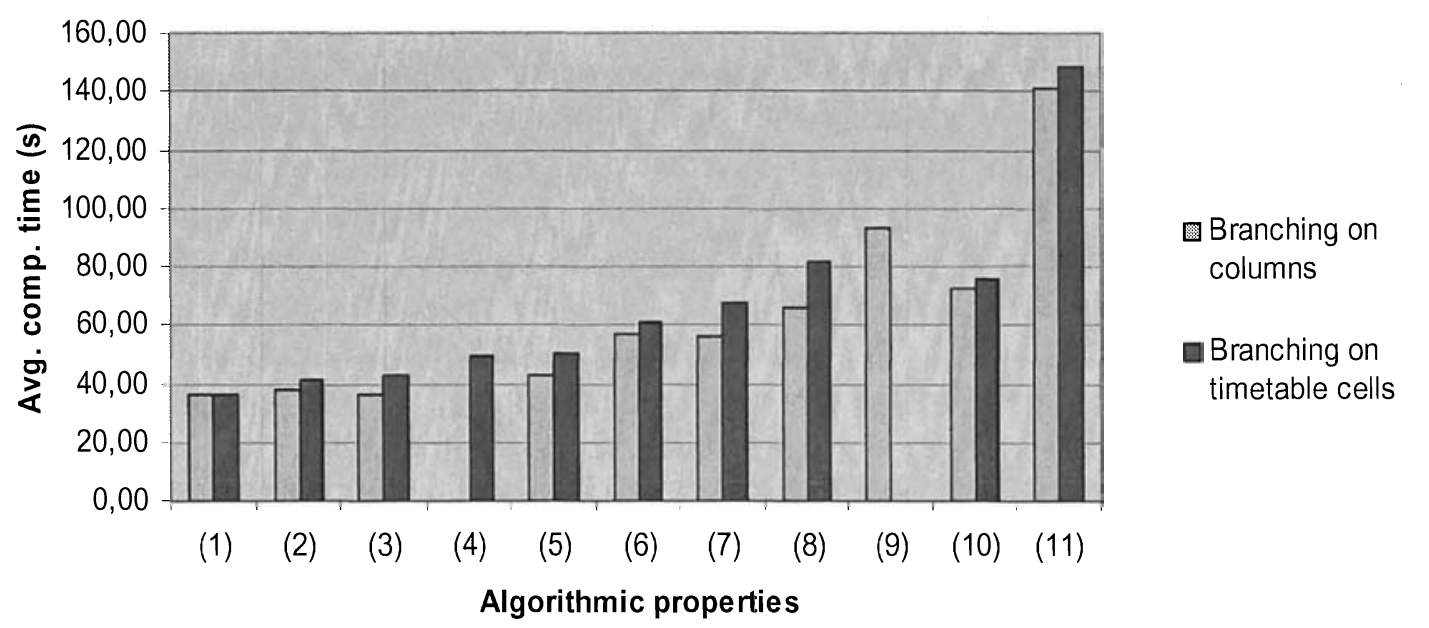

Figure 10: Contributions of algorithmic improvements

\footnotetext{
${ }^{3}$ Subgradient optimization settings include:

- 10 subgradient optimization iterations per simplex iteration;

- 50 iterations of dual price updating within each subgradient optimization iteration;

- new columns priced out based on the optimal dual prices.
} 


\section{Conclusions and future research}

In this paper the problem of scheduling trainees at a hospital department was addressed. In the first part, the problem was stated and formulated as an integer program. Next, a branch-and-bound algorithm was proposed to solve the problem to optimality. The drawbacks of this approach served as a link towards a branch-and-price approach. Therefore, the problem was reformulated as a zero-one multi-commodity flow problem with side-constraints in which we decomposed on the activities. In the next sections the different parts of the branch-and-price algorithm were discussed extensively. The pricing problem could be formulated as a constrained shortest path problem and can be solved efficiently using a forward dynamic programming approach. A very important feature of this dynamic program is the ability to find also the $2^{\text {nd }}, 3^{\text {rd }}$ or $\mathrm{k}^{\text {th }}$ shortest path at a very low computational extra cost. This property enabled us to develop a branching scheme based on the column variables. Alternatively, a branching scheme based on timetable cells and two precedence relations based branching schemes were elaborated. Finally, several speed-up techniques were discussed. In the next part, extensive computational results were presented. An experiment was set up in which the influence of six factors on the complexity of the problem was investigated and the four branching schemes were compared. Concerning theoretical issues, there are two main conclusions. The first one is that the branch-and-price algorithm clearly outperforms the branch-and-bound algorithm. The second is that, within the branch-and-price algorithm, branching on the timetable cells turned out to provide the best results in the most consistent way. Compared to branching on the column variables, this branching scheme is more robust when the magnitude of the non-availability costs contains variability. The branching schemes based on precedence relations converge more slowly to an optimal solution and moreover are not guaranteed to branch until a completely integer solution has been found.

Concerning practical issues, the application makes it possible to find better solutions in less time compared to previous ways of scheduling. To illustrate this, earlier schedules were built for 18 periods. These 18 periods represented 52 weeks (16 3-week periods and 22 -week periods). If a trainee was not available during a certain week, the full period was made unavailable (for scheduling the difficult activities). The developed application is able to deal with scheduling problems for 52 periods. Also, the formerly seniority based division of weeks-off can now be replaced by an approach that takes as much as possible all preferences of all trainees into account. Of course, senior trainees may still be given more priority by assigning them a larger total amount of non-availability costs.

Despite all the improvements, the borders of optimality searching within reasonable time were reached when considering problems starting from twelve trainees and ten activities. Obviously, the exact branch-and-price algorithm can also provide heuristic solutions by allowing a gap between the lower and upper bound. It would, however, be interesting to develop robust heuristic solution procedures that provide good solutions in small computation times. Another interesting research direction would be to adapt the existing branch-and-price algorithm to handle setup costs explicitly. Setup costs would occur each time an assistant (re)starts a certain activity. In this way, one could search for the optimal tradeoff between assigning preferred weeks-off and splitting up activities within trainees. 


\section{Acknowledgements}

We acknowledge the support given to this project by the Fonds voor Wetenschappelijk Onderzoek $(F W O)$ - Vlaanderen, Belgium under contract number G.0463.04. We are grateful to Prof. Dr. W. Sermeus and Kris Vanhaecht of the Centrum voor Ziekenhuis- en Verplegingswetenschap $(C Z V)$ for drawing our attention to challenging scheduling problems in health care management and to Jenny Cristael, (Oogziekenhuis UZ Leuven, Belgium) for providing case study data.

\section{References}

Aickelin, U. and Dowsland, KA., Exploiting problem structure in a genetic algorithms approach to a nurse rostering problem, Journal of Scheduling, Vol. 31, pp. 139-153, 2000.

Barnhart, C., Johnson, E.L., Nemhauser, G.L., Martin, W.P., Savelsbergh, W.P. and Vance P.H., Branch-and-price: column generation for solving huge integer programs, Operations Research, Vol. 46(3), pp. 316-329, 1998.

Beaumont, N., Scheduling staff using mixed integer programming, European Journal of Operational Research, Vol. 98, pp. 473-484, 1997.

Bellman, R., Dynamic Programming, Princeton University Press, 1957.

Brusco, MJ and Jacobs, LW, A simulated annealing approach to the cyclic staffscheduling problem, Naval Research Logistics, Vol. 40, pp. 69-84, 1993.

Bosi, F. and Milano, M., Enhancing constraint logic programming branch and bound techniques for scheduling problems, Software Practice \& Experience, Vol. 31, John Wiley \& Sons, 2001.

Burke, E.K., De Causmaecker, P. and Vanden Berghe, G., A hybrid tabu search algorithm for the nurse rostering problem, in Selected Papers from the 2nd Asia Pacific Conference on Simulated Evolution and Learning, Springer Verlag, Vol. 1585 of LNAI, pp. 187-194, 1998.

Cappanera, P. and Gallo, G., A multi-commodity flow approach to the crew rostering problem, Technical Report, TR-01-08, Dip. di Informatica, Univ. di Pisa, 2001.

Cheang B., Li H., Lim A., Rodrigues B., Nurse rostering problems - A bibliographic survey, European Journal of Operations Research, Vol. 151, pp. 447-460, 2003.

Demeulemeester, E. and Herroelen, W.S., A branch-and-bound procedure for the multiple resource-constrained project scheduling problem, Management Science, Vol. 38(12), pp. 1803-1818, 1992. 
Dreyfus, S.E. and Law, A.M., The art and theory of dynamic programming, Academic Press, Inc. Ltd., 1977.

Hans, E.W., Resource loading by branch-and-price techniques, Twente University Press, Enschede, The Netherlands, pp. 38-42, 2001.

Jans, R., Capacitated lot sizing problems: New applications, formulations and algorithms, Doctoraal Proefschrift, Faculteit Economische en Toegepaste Economische Wetenschappen, 2002.

Jiménez, V.M. and Marzal, A., Computing the K shortest paths: A new algorithm and an experimental comparison, in Lecture Notes in Computer Science Series, J.S. Vitter and C.D. Zaroliagis (eds.), Spinger-Verlag, Vol. 1668, pp. 15-29, 1999.

Mason, A.J. and Smith, M.C., A nested column generator for solving rostering problems with integer programming, in International Conference on Optimisation: Techniques and Applications, L. Caccetta; K. L. Teo; P. F. Siew; Y. H. Leung; L. S. Jennings, and V. Rehbock (eds.), Curtin University of Technology, Perth, Australia, pp. 827-834, 1998.

Mehrotra, A., Murphy, K.E. and Trick, M.A., Optimal shift scheduling: A branch-andprice Approach, Naval Research Logistics, Vol. 47, pp. 185-200, 2000.

Peeters, M., One dimensional cutting and packing: New problems and algorithms, Doctoraal Proefschrift, Faculteit Economische en Toegepaste Economische Wetenschappen, 2002.

Ryan, D.M. and Foster, B.A., An integer programming approach to scheduling, Computer Scheduling of Public Transport Urban Passenger Vehicle and Crew Scheduling, A. Weren (ed.), North-Holland, Amsterdam, pp. 269-280, 1981.

Van den Akker, M., Hoogeveen H. and Van de velde, S.L., Combining column generation and lagrangian relaxation to solve a single-machine common due date problem, INFORMS Journal on Computing, Vol. 14(1), pp. 37-51, 2002.

Vanderbeck, F., On Dantzig-Wolfe decomposition in integer programming and ways to perform branching in a branch-and-price algorithm, Operations Research, Vol. 48(1), pp. 111-128, 2000.

Vanderbeck, F. and Wolsey, L.A., An exact algorithm for IP column generation, Operations Research Letters, Vol. 19, pp. 151-159, 1996.

Warner, M.D., Scheduling nursing personnel according to nurses preference: $A$ mathematical programming approach, Operations Research, Vol. 24(5), pp. 842-856, 1976. 
Appendix 1: Computational results

\begin{tabular}{|c|c|c|c|c|c|c|c|c|c|c|c|c|c|c|c|c|c|}
\hline & & & & $\begin{array}{r}\text { Branch } \\
\mathrm{v}\end{array}$ & $\begin{array}{l}\mathrm{ng} \text { on } \\
\text { ariables }\end{array}$ & olumn & Branchi & $\begin{array}{l}\text { g on ti } \\
\text { cells }\end{array}$ & etable & $\begin{array}{r}\text { Branc } \\
\text { pree }\end{array}$ & $\begin{array}{l}\text { ing on } \\
\text { edence }\end{array}$ & $\begin{array}{l}\text { immedia } \\
\text { relations }\end{array}$ & & & $\begin{array}{l}\text { anching } \\
\text { cedenc }\end{array}$ & $\begin{array}{l}\text { on norme } \\
\text { e relation }\end{array}$ & \\
\hline Problem & Root LP & Heur & Opt & Time & Cols & Nodes & Time & Cols & Nodes & Time & Cols & Nodes & Fract & Time & Cols & Nodes & Fract \\
\hline DOE222111_? & 13 & 14 & 13 & 0.22 & 69 & & 0.2 & 63 & & 0.2 & 63 & 0 & & 0.2 & 63 & 0 & \\
\hline DOE222111_2 & 16 & 20 & 16 & 0.36 & 95 & 3 & 0.28 & 80 & 2 & 0.28 & 83 & 2 & 0 & 0.27 & 79 & 1 & \\
\hline DOE222111_3 & 16 & 17 & 16 & 0.2 & 60 & 1 & 0.25 & 69 & 4 & 0.25 & 65 & 5 & 0 & 0.31 & 78 & 8 & 0 \\
\hline DOE222112_1 & 36 & 41 & 36 & 0.2 & 60 & 0 & 0.25 & 73 & 2 & 0.38 & 104 & 3 & 0 & 0.38 & 107 & 3 & 0 \\
\hline DOE222112_2 & 50 & 53 & 50 & 0.22 & 69 & 0 & 0.28 & 84 & 2 & 0.28 & 89 & 2 & 0 & 0.44 & 116 & 5 & 0 \\
\hline DOE222112_3 & 45 & 45 & 45 & 0.13 & 43 & 0 & 0.11 & 39 & 0 & 0.09 & 39 & 0 & 0 & 0.11 & 39 & 0 & 0 \\
\hline DOE222121_1 & 11 & 12 & 11 & 0.25 & 75 & 0 & 0.28 & 80 & 1 & 0.31 & 90 & 1 & 0 & 0.36 & 91 & 1 & 0 \\
\hline DOE222121_2 & 15 & 19 & 15 & 0.44 & 121 & 2 & 0.28 & 83 & 1 & 0.28 & 81 & 2 & 0 & 0.3 & 83 & 2 & \\
\hline DOE222121_3 & 16 & 17 & 16 & 0.36 & 103 & 3 & 0.41 & 106 & 4 & 0.31 & 88 & 2 & 0 & 0.53 & 130 & 8 & \\
\hline DOE222122_1 & 42 & 44 & 42 & 0.42 & 110 & 4 & 0.38 & 103 & 3 & 0.41 & 108 & 4 & 1 & 0.36 & 96 & 3 & c \\
\hline DOE222122_2 & 33.5 & 43 & 34 & 0.34 & 94 & 1 & 0.33 & 94 & 1 & 0.31 & 90 & 1 & 0 & 0.31 & 90 & 1 & 0 \\
\hline DOE222122_3 & 31 & & 31 & 0.33 & 94 & 0 & 0.33 & 91 & 0 & 0.31 & 91 & 0 & 0 & 0.33 & 91 & 0 & 0 \\
\hline DOE222131_1 & 10 & 14 & 10 & 0.63 & 154 & 3 & 0.64 & 167 & 2 & 0.58 & 143 & 2 & 0 & 0.5 & 133 & 1 & 0 \\
\hline DOE222131_2 & 13.5 & 16 & 14 & 0.34 & 95 & 0 & 0.55 & 138 & 4. & 0.67 & 163 & 6 & 0 & 0.55 & 136 & 4 & \\
\hline DOE222131_3 & 10.66 & 16 & 12 & 2.91 & 480 & 28 & 1.64 & 334 & 12] & 1.45 & 295 & 9 & 0 & 1.91 & 359 & 17 & \\
\hline DOE222132_1 & 35.75 & 45 & 37 & 3.17 & 404 & 60 & 0.98 & 216 & 9 & 1.38 & 278 & 15 & 0 & 0.72 & 156 & 8 & \\
\hline DOE222132_2 & 33.5 & 50 & 35 & 1.03 & 218 & 7 & 1.01 & 223 & 6 & 1.05 & 224 & 8 & 0 & 1.06 & 224 & 8 & c \\
\hline DOE222132_3 & 31.5 & & 33 & 1.88 & 340 & 18 & 1.3 & 312 & 8 & 1.89 & 413 & 9 & 0 & 1.98 & 360 & 16 & 1 \\
\hline DOE222:41_1 & 9.56 & 13 & 10 & 0.83 & 197 & 2 & 0.95 & 214 & 7 & 0.98 & 221 & 5 & 0 & 1.16 & 242 & 8 & 0 \\
\hline DOE222141_2 & 12.3 & & 13 & 0.72 & 161 & 5 & 1.05 & 222 & 12 & 0.97 & 215 & 7 & 0 & 1.23 & 256 & 9 & 0 \\
\hline DOE222141_3 & 13.33 & - & 14 & 0.42 & 112 & 1 & 0.69 & 156 & 6 & 0.66 & 155 & 5 & 0 & 0.59 & 133 & 5 & 0 \\
\hline DOE222142_1 & 28.83 & 45 & 31 & 2.81 & 433 & 25 & 2.25 & 419 & 16 & 2.22 & 421 & 16 & ol & 4.4 & 712 & 38 & \\
\hline DOE222 & 30.8 & 40 & 32 & 2.17 & 380 & 19 & 2.13 & 401 & 16 & 2.14 & 388 & 16 & 0 & 2.23 & 397 & 15 & c \\
\hline DOE222142_3 & 22.1 & 25 & 24 & 8.47 & 705 & 120 & 2.38 & 425 & 20 & 2.77 & 488 & 21 & 0 & 4.03 & 652 & 29 & 0 \\
\hline DOE222151_1 & 14 & 16 & 14 & 0.38 & 100 & 2 & 0.77 & 183 & 5 & 0.75 & 182 & 5 & 0 & 0.59 & 138 & 7 & c \\
\hline DOE222151_2 & 13.38 & 16 & 14 & 0.55 & 134 & 2 & 0.56 & 143 & 3 & 0.73 & 168 & 5 & 0 & 0.73 & 159 & 6 & c \\
\hline DOE222151_3 & 13.33 & 16 & 14 & 0.41 & 105 & 2 & 0.47 & 114 & 6 & 0.42 & 109 & 4 & 0 & 0.56 & 128 & 9 & c \\
\hline DOE222152_1 & 43 & 54 & 43 & 0.25 & 74 & 1 & 0.45 & 116 & 2 & 0.42 & 113 & 1 & 0 & 0.47 & 119 & 1 & c \\
\hline DOE222 & 42 & 49 & 43 & 3 & 469 & 41 & 1.58 & 312 & 15 & & & 11 & 1 & 1.5 & 288 & 13 & \\
\hline DOE22 & 37 & 44 & 37 & 0.34 & 95 & 1 & 0.33 & 94 & & 0.3 & 93 & 0 & 0 & 0.33 & 93 & 0 & \\
\hline DOE222161_1] & 12 & 14 & 12 & 0.86 & 198 & 3 & 0.8 & 189 & 4 & 0.63 & 150 & 2 & 0 & 0.66 & 147 & 4 & \\
\hline DOE222161_2 & 10.63 & 13 & 11 & 0.88 & 192 & 4 & 0.72 & 166 & 5 & 0.97 & 211 & 5 & 0 & 1.44 & 282 & 10 & \\
\hline DOE222161_3 & 12.5 & 14 & 13 & 0.78 & 176 & 7 & 0.45 & 119 & 3 & 0.69 & 172 & 4 & 0 & 0.45 & 113 & 3 & \\
\hline DOE222162_1 & 32 & 47 & 32 & 0.56 & 146 & 0 & 0.77 & 191 & 2 & 0.83 & 201 & 2 & 0 & 0.8 & 194 & 1 & c \\
\hline DOE222162_2 & 37 & 45 & 37 & 0.39 & 106 & 0 & 0.44 & 120 & 0 & 0.44 & 120 & 0 & 0 & 0.44 & 120 & 0 & c \\
\hline DOE22216 & 33.58 & 43 & 35 & 1.64 & 349 & 8 & 1.64 & 341 & 7 & & & 7 & I & 2. & 454 & 10 & \\
\hline & 13 & 14 & 13 & 1.03 & 227 & 4 & 1.53 & 325 & 14 & 1.31 & 267 & 10 & & 2.05 & 336 & 23 & \\
\hline DOE222211_2 & 14 & 14 & 14 & 0.69 & 153 & 6 & 0.31 & 90 & 0 & 0.3 & 84 & 0 & 0 & 0.3 & 84 & 0 & \\
\hline DOE222211_3 & 13 & 16 & 13 & 1.34 & 281 & 7 & 1.36 & 286 & 1 & 1.59 & 317 & 7 & 0 & 1.84 & 319 & 17 & \\
\hline DOE222212_1 & 33 & 41 & 33 & 0.88 & 210 & 3 & 1.42 & 299 & 12 & 1.38 & 286 & 11 & 0 & 1.8 & 342 & 16 & \\
\hline DOE222212_2 & 31 & 36 & 31 & 0.34 & 90 & 0 & 1.3 & 273 & 12 & 1.36 & 295 & 10 & 0 & 1.73 & 297 & 24 & \\
\hline DOE222212_3 & 26 & 32 & 26 & 1.06 & 246 & 5 & 1.63 & 352 & 12 & 1.53 & 309 & 10 & 0 & 1.88 & 327 & 19 & \\
\hline & 6 & 9 & 6 & 3.39 & 507 & 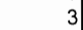 & 7.61 & 1025 & 1 & & & 9 & & 15.5 & 1271 & 16 & \\
\hline & 9 & 12 & 9 & 2.11 & 315 & ? & & 526 & 10 & 5.6 & & 10 & & $5 . \varepsilon$ & 637 & 12 & \\
\hline DOE222 & 8 & 15 & 8 & 2.59 & 385 & 3 & 3.39 & 507 & & 4.25 & 603 & 7 & & 3.91 & 422 & 11 & \\
\hline DOE222 & 26 & 35 & 26 & 4.38 & 656 & 0 & 4.69 & 691 & 4 & 4.02 & 562 & 6 & 0 & 5.98 & 634 & 14 & \\
\hline DOE222222_2 & 31.57 & 42 & 33 & 157.94 & 3844 & 290 & 61.45 & 2984 & 141 & 23.72 & 1732 & 39 & 0 & 74.88 & 2831 & 147 & \\
\hline DOE222222_3 & 18 & 27 & 18 & 7.75 & 1005 & 11 & 3. & 534 & 3 & 3.66 & 522 & 2 & 0 & 5.19 & 576 & 5 & \\
\hline DOE222 & 6 & 12 & 6 & 3.4 & 432 & 1 & 6.99 & 737 & 6 & 19.88 & 1417 & 18 & ol & 20.34 & 1211 & 18 & \\
\hline DOE22: & 6 & 10 & 7 & 123.25 & 3854 & 130 & 45.22 & 2791 & 45 & 42.8 & 249 & 38 & & 61.94 & 2585 & 67 & \\
\hline & 6 & 9 & 6 & 3.2 & 33 & & 4. & & & 5.7 & & 2 & & 9.7 & 745 & 5 & \\
\hline & 17 & 27 & 18 & 361.89 & 8807 & 354 & 119.61 & 4431 & 111 & 66 & 2947 & 63 & & 110.95 & 3738 & 89 & \\
\hline DOE22 & 15.1 & 21 & 16 & 9.59 & 1062 & 7 & 78.73 & 3639 & 100 & 14.13 & 1172 & 15 & 0 & 16.53 & 1072 & 21 & \\
\hline & 15 & 25 & 15 & & 71 & 5 & & 79 & 11 & 26. & 1840 & 22 & 0 & 35.47 & 1819 & 31 & \\
\hline & 7 & 9 & 7 & 5.8 & 581 & 20 & & 73 & 12 & 7.1 & 755 & 16 & 象 & 7.2 & 474 & 20 & \\
\hline DOE22 & 8 & 9 & 8 & 4.13 & 376 & 3 & 6.56 & 652 & 16 & & & 18 & 田 & 12.2 & 697 & 32 & \\
\hline DOE22 & 5 & 9 & 5 & 15.3 & 128 & 19 & 7.0 & 67 & & 8. & & 7 & & 25.9 & 1224 & 18 & \\
\hline & 18 & 25 & 18 & 57.14 & 325 & 46 & 11.02 & 100 & & 89.0 & 312 & 61 & & 67.38 & 2306 & 29 & \\
\hline & 8 & 24 & 8 & 8 & 717 & 5 & 8. & 97 & 7 & 13. & 10 & 11 & & 16. & & 9 & \\
\hline $\mathrm{DOE}$ & 11 & 34 & 11 & 10.52 & 75 & 2 & & 7 & 2 & 19. & 125 & 6 & 0 & 34. & 1553 & 11 & \\
\hline & 8.1 & 12 & 9 & & 43 & 4 & & & 4 & & 671 & 8 & 田 & 11.2 & 1035 & 28 & \\
\hline DOE22225 & 9 & 13 & 10 & & 973 & 15 & 37.73 & 2540 & 69 & 273.53 & 4984 & 252 & 0 & $>600$ & 7134 & 506 & \\
\hline DOE222251_3 & 6.51 & 13 & 7 & 9.08 & 1063 & 50 & 29.54 & 2015 & 50 & & 1193 & 16 & 0 & 25.47 & 1515 & 30 & \\
\hline DOE222252_1 & 10.81 & 27 & 13 & $>600$ & 13252 & 1163 & 453.45 & 10680 & 684 & 374.5 & & 465 & 0 & 561.53 & 10154 & 592 & \\
\hline & 22. & 38 & 24 & & & 14 & 9. & & 1 & & & 2 & 0 & & & 58 & \\
\hline & 19.49 & 29 & 22 & 512 & & 58 & & & 211 & & & 225 & 0 & 171.3 & 491 & 192 & \\
\hline & 6 & 11 & 6 & & & & & & 20 & 12. & 100 & 10 & o & 11.47 & 731 & 16 & \\
\hline DOE222261_2 & 7 & 10 & 8 & 121.47 & 3804 & 127 & 36.12 & 2485 & 44 & 57.07 & 2936 & 59 & 0 & 35.17 & 1759 & 42 & \\
\hline DOE222261_3 & 4.01 & 10 & 5 & 35.36 & 2033 & 108 & 30.5 & 2425 & 44 & 7.91 & 719 & 10 & 0 & 10.5 & 693 & 23 & \\
\hline DOE222262_1 & 14.14 & 38 & 15 & & 86 & 7 & & 99 & 12 & & 13 & 21 & & & 10 & 15 & \\
\hline & 20.2 & 27 & 21 & & & 15 & & 21 & & & & 32 & 0 & & 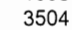 & 74 & \\
\hline & 13.98 & 28 & 16 & 344. & 7687 & 1198 & 113. & 5798 & 243 & 184. & 569 & 274 & 0 & 193.96 & 6041 & 312 & \\
\hline & 15 & 15 & 15 & 0. & 70 & 0 & 0. & 87 & 0 & 0.2 & 77 & 0 & 0 & 0.31 & 77 & 0 & \\
\hline DOE222311_2 & 15 & 15 & 15 & 0.42 & 95 & 0 & 0.34 & 89 & d & 0.34 & 89 & 0 & 0 & 0.36 & 89 & 0 & \\
\hline DOE222311_3 & 12 & 13 & 12 & 0.41 & 96 & 0 & 1.19 & 238 & 15 & 1.28 & 252 & 14 & 0 & 1.31 & 193 & 17 & \\
\hline DOE222312_1 & 37 & 37 & 37 & 0.55 & 125 & 0 & 0.36 & 89 & 0 & 0.3 & 100 & 0 & & 0.4 & 100 & 0 & \\
\hline & 34 & 39 & 34 & & 90 & 0 & & 300 & 15 & 1 & & 14 & & & 327 & 32 & \\
\hline OF?23231 & 40 & 40 & 40 & 0.52 & 117 & 0 & 0.37 & 96 & 0 & 0.39 & 100 & 0 & & 0.42 & 100 & 0 & \\
\hline
\end{tabular}




\begin{tabular}{|c|c|c|c|c|c|c|c|c|c|c|c|c|c|c|c|}
\hline & & & & Branch & $\begin{array}{l}\text { ing on } \\
\text { lariable }\end{array}$ & olumn & Branch & $\begin{array}{l}\text { ng on ti } \\
\text { cells }\end{array}$ & petable & $\begin{array}{r}\text { Bran } \\
\text { pre }\end{array}$ & $\begin{array}{l}\text { hing on } \\
\text { cedence }\end{array}$ & $\begin{array}{l}\text { immedia } \\
\text { relations }\end{array}$ & & & $\begin{array}{l}\text { anching } \\
\text { ecedenc }\end{array}$ \\
\hline Problem & Root LP & Heur & Opt & Time & Cols & Nodes & Time & Cols & Nodes & Time & Cols & Nodes & Fract & Time & Cols \\
\hline DOE222321_1 & 6 & 9 & 6 & 6.78 & 561 & 2 & 15.27 & 7412 & 15 & 12.15 & 910 & 18 & 0 & 21.52 & 1076 \\
\hline DOE222321_2 & 5 & 11 & 5 & 7.25 & 642 & 2 & 18.13 & 1380 & 11 & 9.11 & 628 & 7 & 0 & 122.44 & 3554 \\
\hline DOE222321_3 & 8 & 11 & 8 & 5.69 & 527 & 4 & 15.33 & 1429 & 18 & 10.83 & 880 & 15 & 1 & 19.6 & 1068 \\
\hline DOE222322_1 & 14 & 24 & 14 & 24.9 & 1555 & 7 & 18.8 & 1409 & 7 & 41.35 & 1886 & 17 & 0 & 69.25 & 2114 \\
\hline DOE222322_2 & 18 & 28 & 18 & 10.14 & 820 & 6 & 13.85 & 1167 & 13 & 12.97 & 839 & 7 & 0 & 24.9 & 948 \\
\hline DOE222322_3 & 10 & 26 & 10 & 12.77 & 1283 & 16 & 6.6 & 805 & 6 & 6.72 & 755 & 11 & 0 & 27.45 & 1664 \\
\hline DOE222331_1 & 5 & 8 & 5 & 8.49 & 501 & 2 & 14.74 & 1182 & 18 & 15.2 & 705 & 10 & 0 & 52.66 & 1432 \\
\hline DOE222331_2 & 5 & 9 & 5 & 13.85 & 916 & 5 & 13.55 & 1251 & 16 & 20.78 & 1236 & 18 & 0 & 27.13 & 827 \\
\hline DOE222331_3 & 6 & 9 & 6 & 10.02 & 664 & 5 & 16.02 & 1112 & 15 & 29.52 & 1324 & 17 & 0 & 140.12 & 3406 \\
\hline DOE222332_1 & 17 & 26 & 17 & 14.2 & 831 & 6 & 10.78 & 969 & 9 & 13.13 & 999 & 18 & 0 & 31.7 & 1134 \\
\hline DOE222332_2 & 12 & 24 & 12 & 14 & 772 & 5 & 12.5 & 902 & 8 & 18.78 & 1011 & 10 & 0 & 42.2 & 1425 \\
\hline DOE222332_3 & 8.6 & 20 & 9 & 29.31 & 1586 & 20 & 90.05 & 3384 & 58 & 29.16 & 1532 & 15 & 0 & 58.11 & 1757 \\
\hline DOE222341_1 & 4 & 6 & 4 & 12.44 & 494 & 2 & 25.91 & 1159 & 19 & 32.47 & 1052 & 16 & 0 & 67.01 & 1109 \\
\hline DOE222341_2 & 5 & 8 & 5 & 18.72 & 777 & 4 & 18.81 & 1281 & 17 & 32.16 & 1404 & 25 & 0 & 104.15 & 1716 \\
\hline DOE222341_3 & 2 & 6 & 2 & 13.56 & 567 & 2 & 14.45 & 891 & 10 & 27.3 & 966 & 9 & 0 & 43.86 & 925 \\
\hline DOE222342_1 & 13 & 19 & 13 & 24.11 & 1015 & 14 & 24.59 & 1443 & 18 & 22.13 & 851 & 11 & 0 & 50.77 & 929 \\
\hline DOE222342_2 & 4 & 11 & 4 & 40.08 & 1455 & 27 & 29.33 & 1467 & 16 & 34.59 & 1226 & 16 & 0 & 77.47 & 1383 \\
\hline DOE222342_3 & 4 & 17 & 4 & 20.31 & 827 & 4 & 19.11 & 1075 & 12 & 32.99 & 1178 & 14 & 0 & 49.38 & 1077 \\
\hline DOE222351_1 & 5 & 8 & 5 & 11.72 & 677 & 3 & 18.81 & 1442 & 16 & 9.48 & 772 & 12 & 0 & 13.91 & 786 \\
\hline DOE222351_2 & 9.37 & 12 & 10 & 20.89 & 1424 & 34 & 15.05 & 1378 & 24 & 20.44 & 1351 & 14 & 0 & 378.05 & 6198 \\
\hline DOE222351_3 & 5.69 & 10 & 6 & 165.3 & 4671 & 255 & 18.7 & 1236 & 15 & 46.28 & 1819 & 32 & 0 & 225.8 & 3486 \\
\hline DOE222352_1 & 10.83 & 19 & 11 & 14.22 & 1144 & 9 & 15.89 & 1257 & 11 & 71.78 & 2520 & 41 & 0 & 150.34 & 3472 \\
\hline DOE222352_2 & 18.29 & 27 & 19 & $>600$ & 9249 & 623 & 247.14 & 5900 & 99 & 256.78 & 5050 & 104 & 0 & $>600$ & 6964 \\
\hline DOE222352_3 & 18 & 24 & 18 & 9.84 & 703 & 9 & 8.16 & 646 & 7 & 36.75 & 1341 & 18 & 0 & 160.02 & 2938 \\
\hline DOE222361_1 & 7 & 10 & 7 & 14.08 & 649 & 4 & 16.64 & 933 & 12 & 24.82 & 919 & 11 & 0 & 55.21 & 1077 \\
\hline DOE222361_2 & 5 & 7 & 5 & 23.05 & 1056 & 13 & 14.8 & 810 & 7 & $>600$ & 7174 & 102 & 0 & 259.96 & 3616 \\
\hline DOE222361_3 & 5 & 9 & 5 & 65.8 & 2606 & 38 & $>600$ & 9508 & 173 & 393.65 & 7522 & 132 & 0 & 298.31 & 4419 \\
\hline DOE222362_1 & 8 & 19 & 8 & 34.73 & 1681 & 23 & 38.97 & 2354 & 29 & 47.78 & 1855 & 17 & 0 & 39.08 & 1012 \\
\hline DOE222362_2 & 15 & 26 & 15 & 83.53 & 3091 & 20 & 14.13 & 1046 & 8 & 61.13 & 2432 & 18 & 0 & 97.81 & 2619 \\
\hline DOE222362_3 & 6 & 15 & 7 & 234.16 & 6940 & 273 & $>600$ & 10322 & 226 & $>600$ & 7921 & 155 & 0 & $>600$ & 7615 \\
\hline DOE222411_1 & 12.25 & - & 15 & $>600$ & 11797 & 3031 & 129.77 & 5981 & 515 & $>600$ & 7214 & 1159 & 0 & $>600$ & 7502 \\
\hline DOE222411_2 & 10.78 & - & - & $>600$ & 12467 & 1856 & $>600$ & 13338 & 2064 & $>600$ & 7322 & 1200 & 0 & $>600$ & 7991 \\
\hline DOE222411_3 & 10.64 & - & & $>600$ & 10479 & 1409 & $>600$ & 12713 & 1941 & $>600$ & 9326 & 1304 & 0 & $>600$ & 8044 \\
\hline DOE2224.12_1 & 26.43 & - & 29 & 53.36 & 2955 & 328 & 24.56 & 2436 & 94 & 19.05 & 1997 & 59 & 0 & 37.77 & 2641 \\
\hline DOE222412_2 & 24.5 & - & 28 & 11.86 & 1447 & 70 & 10.02 & 1266 & 65 & 26.86 & 2226 & 189 & 1 & 83.03 & 3911 \\
\hline DOE222412_3 & 32.25 & - & & $>600$ & 12525 & 3968 & $>600$ & 17638 & 3646 & $>600$ & 12019 & 2860 & 0 & $>600$ & 13329 \\
\hline DOE222421_1 & 6.57 & 12 & 7 & 9.97 & 1216 & 27 & 21.72 & 1928 & 33 & 53.23 & 2848 & 46 & 0 & 7.27 & 649 \\
\hline DOE222421_2 & 7.4 .1 & 13 & 8 & 4.5 & 460 & 3 & 6.69 & 687 & 13 & 8.45 & 745 & 10 & 0 & 13.23 & 842 \\
\hline DOE222421_3 & 8 & 10 & 8 & 13.59 & 1119 & 37 & 5.89 & 622 & 8 & 6.14 & 502 & 4 & 0 & 12.84 & 626 \\
\hline DOE222422_1 & 13 & 29 & - & $>600$ & 12668 & 864 & $>600$ & 13691 & 452 & $>600$ & 11584 & 357 & 0 & $>600$ & 11748 \\
\hline DOE222422_2 & 17.6 & 29 & 20 & 394.74 & 8729 & 425 & 75.22 & 4610 & 66 & 154.2 & 5828 & 90 & 0 & 364.47 & 7436 \\
\hline DOE222422_3 & 12.29 & 25 & 13 & 8.31 & 928 & 10 & 14.09 & 1336 & 11 & 8.86 & 809 & 5 & 0 & 12.28 & 755 \\
\hline DOE222431_1 & 5 & 7 & 6 & 25.14 & 1352 & 17 & 15.44 & 1241 & 14 & 41.98 & 1892 & 29 & 1 & 31.03 & 1162 \\
\hline DOE222431_2 & 4 & 10 & 4 & 4.7 & 405 & 1 & 9.42 & 780 & 5 & 7 & 554 & 2 & 0 & 9.09 & 530 \\
\hline DOE222431_3 & 4.89 & 9 & 6 & 19.42 & 1098 & 14 & 14 & 960 & 14 & 22.44 & 1184 & 15 & 0 & 53.45 & 1787 \\
\hline DOE222432 1 & 9.7 & 19 & 12 & 64.34 & 2693 & 90 & 77.61 & 4047 & 76 & 46.56 & 2610 & 36 & 0 & 71.06 & 2707 \\
\hline DOE222432_2 & 20.49 & 33 & 21 & 7.44 & 564 & 3 & 8.16 & 607 & 6 & 9.33 & 606 & 5 & 0 & 18.19 & 745 \\
\hline DOE222432_3 & 10 & 23 & 10 & 8.86 & 528 & 1 & 5.89 & 553 & 2 & 6.78 & 576 & 2 & 0 & 7.92 & 498 \\
\hline DOE222441 1 & 5.04 & 8 & 6 & 29.3 & 1489 & 20 & 27.94 & 1835 & 36 & 41.39 & 1824 & 17 & 0 & 54.14 & 1550 \\
\hline DOE222441_2 & 2 & 5 & 2 & 14.69 & 746 & 4 & 11.06 & 654 & 3 & 18.14 & 815 & 6 & 0 & 19.89 & 592 \\
\hline DOE222441_3 & 5 & 7 & 5 & 22.3 & 1105 & 13 & 15.2 & 1120 & 15 & 12.19 & 775 & 11 & 0 & 22.81 & 975 \\
\hline DOE222442 1 & 3 & 12 & 3 & 28.92 & 1077 & 7 & 16.11 & 925 & 3 & 39.25 & 1640 & 12 & 0 & 72.5 & 1731 \\
\hline DOE222442_2 & 9 & 16 & 9 & 14.83 & 721 & 2 & 35.8 & 1743 & 10 & 45.14 & 1564 & 6 & 0 & 48.42 & 1321 \\
\hline DOE222442_3 & 12 & 21 & 13 & 69.5 & 2487 & 80 & 26.83 & 1754 & 12 & 26.88 & 1498 & 13 & 0 & 115.6 & 3603 \\
\hline DOE222451_1 & 6.78 & 12 & 8 & 23.44 & 1822 & 42 & 268.03 & 6138 & 287 & 8.63 & 800 & 10 & 1 & 223.12 & 5367 \\
\hline DOE222451_2 & 7.98 & 12 & 10 & $>600$ & 5747 & 447 & 340.81 & 9565 & 374 & 601 & 11059 & 580 & 0 & $>600$ & 11060 \\
\hline DOE222451_3 & 9.56 & - & 11 & $>600$ & 4990 & 556 & 99.52 & 4264 & 167 & 220.3 & 5614 & 289 & 0 & 424.22 & 7684 \\
\hline DOE222452 1 & 14.56 & 35 & 19 & 254.66 & 8220 & 481 & 140 & 7243 & 140 & 211.41 & 8606 & 181 & 0 & 242.19 & 8003 \\
\hline DOE222452_2 & 14.83 & 33 & 16 & 15.27 & 1079 & 9 & 12.88 & 1073 & 12 & 14.59 & 1089 & 8 & 1 & 25.8 & 1310 \\
\hline DOE222452_3 & 22 & 36 & 25 & 344.08 & 10188 & 727 & 245.36 & 9327 & 258 & $>600$ & 13211 & 396 & 0 & $>600$ & 13195 \\
\hline DOE222461 1 & 5.19 & 9 & 7 & 565.67 & 7296 & 586 & 152.07 & 4598 & 111 & 230.35 & 5295 & 109 & 0 & $>600$ & 6326 \\
\hline DOE222461_2 & 7 & 10 & 7 & 7.94 & 425 & 4 & 105 & 3253 & 55 & 31.23 & 934 & 6 & 0 & 65.44 & 1204 \\
\hline DOE222461_3 & 4 & 7 & 4 & 38.48 & 2042 & 23 & 18.27 & 1206 & 6 & 9.97 & 643 & 4 & 0 & 17.45 & 699 \\
\hline DOE222462 1 & 10 & 18 & 10 & 4.22 & 413 & 2 & 3.41 & 412 & 3 & 7.06 & 680 & 7 & 0 & 10.2 & 724 \\
\hline DOE222462_2 & 14.65 & 37 & 18 & $>600$ & 11515 & 564 & 327 & 10757 & 250 & 515.46 & 12880 & 340 & 0 & $>600$ & 10379 \\
\hline DOE222462_3 & 11.56 & 25 & 13 & 86.82 & 3838 & 91 & 86.05 & 3867 & 47 & 79.45 & 2910 & 38 & 0 & 152.89 & 3269 \\
\hline DOE111231_1 & 1 & 1 & 1 & 0.03 & 7 & 0 & 0.06 & 14 & 0 & 0.06 & 14 & 0 & 0 & 0.06 & 14 \\
\hline DOE111231_2 & 6 & 6 & 6 & 0.03 & 6 & 0 & 0.02 & 6 & 0 & 0.01 & 6 & 0 & 0 & 0.02 & 6 \\
\hline DOE111231_3 & 0 & 0 & 0 & 0 & 3 & 0 & 0 & 3 & 0 & 0 & 3 & 0 & 0 & 0 & 3 \\
\hline DOE111232_1 & 1 & 3 & 1 & 0.09 & 18 & 0 & 0.11 & 20 & 0 & 0.11 & 20 & 0 & 0 & 0.11 & 20 \\
\hline DOE111232_2 & 1 & 2 & 2 & 0.36 & 48 & 5 & 0.3 & 38 & 3 & 0.23 & 29 & 2 & 0 & 0.22 & 29 \\
\hline DOE111232_3 & 8 & 8 & 8 & 0.05 & 10 & 0 & 0.03 & 8 & 0 & 0.03 & 8 & 0 & 0 & 0.03 & 8 \\
\hline DOE112231_1 & 3 & 4 & 3 & 0.14 & 34 & 0 & 0.22 & 47 & 2 & 0.25 & 55 & 2 & 0 & 0.25 & 58 \\
\hline DOE112231_2 & 3 & 7 & 3 & 0.34 & 89 & 0 & 0.28 & 77 & 0 & 0.28 & 77 & 0 & 0 & 0.28 & 77 \\
\hline DOE112231_3 & 4 & 5 & 4 & 0.17 & 50 & 0 & 0.47 & 120 & 4 & 0.36 & 88 & 4 & 0 & 0.45 & 116 \\
\hline DOE112232_1 & 9.25 & 18 & 10 & 0.38 & 94 & 1 & 0.48 & 111 & 3 & 0.63 & 136 & 5 & 0 & 0.44 & 102 \\
\hline DOE112232_2 & 13 & 16 & 13 & 0.09 & 27 & 0 & 0.3 & 70 & 3 & 0.47 & 96 & 4 & 0 & 0.41 & 92 \\
\hline DOE112232_3 & 11 & 17 & 11 & 0.25 & 68 & 0 & 0.23 & 64 & 0 & 0.34 & 88 & 1 & 0 & 0.33 & 90 \\
\hline DOE113231_1 & 8 & - & 8 & 0.45 & 127 & 1 & 0.83 & 215 & 3 & 0.69 & 187 & 3 & 0 & 1.59 & 330 \\
\hline DOE113231_2 & 7 & 9 & 7 & 0.72 & 179 & 3 & 0.73 & 192 & 4 & 1.05 & 250 & 6 & 0 & 0.88 & 222 \\
\hline DOE113231_3 & 8 & 9 & 8 & 0.58 & 165 & 1 & 0.55 & 156 & 1 & 0.91 & 230 & 5 & 0 & 0.95 & 228 \\
\hline
\end{tabular}




\begin{tabular}{|c|c|c|c|c|c|c|c|c|c|c|c|c|c|c|c|}
\hline & & & & $\begin{array}{r}\text { Branch } \\
\mathrm{V}\end{array}$ & $\begin{array}{l}\text { ing on } \\
\text { ariable }\end{array}$ & olumn & Branch & $\begin{array}{l}\text { g on tin } \\
\text { cells }\end{array}$ & netable & $\begin{array}{r}\text { Branc } \\
\text { prec }\end{array}$ & $\begin{array}{l}\text { hing on } \\
\text { edence }\end{array}$ & $\begin{array}{l}\text { immedia } \\
\text { relations }\end{array}$ & & & $\begin{array}{l}\text { nching } \\
\text { cedenc }\end{array}$ \\
\hline Problem & Root LP & Heur & Opt & Time & Cols & Nodes & Time & Cols & Nodes & Time & Cols & Nodes & Fract & Time & Cols \\
\hline DOE113232_1 & 16 & 22 & 16 & 0.58 & 165 & 0 & 0.92 & 243 & 2 & 0.73 & 196 & 1 & of & 0.73 & 196 \\
\hline DOE113232_2 & 21.67 & - & 22 & 1.19 & 298 & 3 & 1.06 & 289 & 3 & 0.92 & 253 & 3 & 0 & 1.44 & 339 \\
\hline DOE113232_3 & 15 & - & 15 & 0.53 & 155 & 0 & 0.58 & 157 & 1 & 0.58 & 156 & 2 & 0 & 0.63 & 156 \\
\hline DOE121231_1 & 0 & 0 & 0 & 0 & 4 & 0 & 0 & 4 & 0 & 0 & 4 & 0 & 0 & 0 & 4 \\
\hline DOE121231_2 & 0 & 0 & 0 & 0 & 4 & 0 & 0 & 4 & 0 & 0 & 4 & 0 & 0 & 0 & 4 \\
\hline DOE121231_3 & 0 & 0 & 0 & 0 & 4 & 0 & 0 & 4 & 0 & 0 & 4 & 0 & 0 & 0 & 4 \\
\hline DOE121232_1 & 0 & 0 & 0 & 0 & 4 & 0 & 0 & 4 & 0 & 0 & 4 & 0 & 0 & 0 & 4 \\
\hline DOE121232_2 & 5 & 5 & 5 & 0.11 & 20 & 0 & 0.13 & 27 & 0 & 0.11 & 26 & 0 & 0 & 0.13 & 26 \\
\hline DOE121232_3 & 0 & 0 & 0 & 0 & 4 & 0 & 0 & 4 & 0 & 0 & 4 & 0 & 0 & 0 & 4 \\
\hline DOE122231_1 & 3 & 4 & 3 & 2.81 & 430 & 5 & 3.88 & 614 & 13 & 4.06 & 538 & 12 & 0 & 6.24 & 616 \\
\hline DOE122231_2 & 2 & 5 & 2 & 2.61 & 430 & 3 & 3.31 & 548 & 10 & 3.89 & 609 & 10 & 0 & 12.45 & 1248 \\
\hline DOE122231_3 & 1 & 3 & 1 & 1.38 & 240 & 2 & 2.64 & 497 & 6 & 3.7 & 525 & 12 & 0 & 15.11 & 1468 \\
\hline DOE122232_1 & 9 & 17 & 9 & 3.11 & 475 & 4 & 3.27 & 550 & 11 & 3.67 & 603 & 13 & 0 & 6.44 & 633 \\
\hline DOE122232_2 & 8 & 15 & 8 & 1.72 & 261 & 3 & 1.88 & 322 & 7 & 3.91 & 566 & 12 & 0 & 5.63 & 617 \\
\hline DOE122232_3 & 7 & 9 & 7 & 4.03 & 547 & 6 & 4.77 & 671 & 15 & 5.06 & 585 & 10 & 0 & 8.89 & 732 \\
\hline DOE123231_1 & 11 & - & 11 & 4.94 & 639 & 9 & 9.27 & 1067 & 20 & 9.2 & 899 & 20 & 0 & 24.72 & 1584 \\
\hline DOE123231_2 & 11 & 15 & 11 & 4.61 & 619 & 4 & 9.38 & 1277 & 20 & 23.48 & 1852 & 38 & 0 & 18.63 & 1246 \\
\hline DOE123231_3 & 9 & 14 & 9 & 4.63 & 674 & 4 & 10.23 & 1332 & 21 & 10.95 & 1088 & 20 & 0 & 19.17 & 1253 \\
\hline DOE123232_1 & 30 & 39 & 30 & 5.75 & 769 & 3 & 10.77 & 1277 & 23 & 9.23 & 995 & 14 & 0 & 15.25 & 1038 \\
\hline DOE123232_2 & 28 & - & 28 & 7.45 & 922 & 6 & 12.08 & 1422 & 19 & 10.83 & 1070 & 16 & 0 & 29.75 & 1822 \\
\hline DOE123232_3 & 26 & 38 & 26 & 9.08 & 1180 & 7 & 15.66 & 2052 & 23 & 15.66 & 1440 & 17 & 0 & 19.77 & 1405 \\
\hline DOE131231_1 & 0 & 0 & 0 & 1.61 & 145 & 3 & 0 & 6 & 0 & 0 & 6 & 0 & 0 & 0 & 6 \\
\hline DOE131231_2 & 1 & 1 & 1 & 0.48 & 50 & 0 & 0.34 & 66 & 0 & 0.34 & 65 & 0 & 0 & 0.42 & 65 \\
\hline DOE131231_3 & 0 & 0 & 0 & 0 & 6 & 0 & 0 & 6 & 0 & 0 & 6 & 0 & 0 & 0 & 6 \\
\hline DOE 131232_1 & 2 & 2 & 2 & 0.88 & 89 & 0 & 0.31 & 76 & 0 & 0.28 & 72 & 0 & 0 & 0.3 & 72 \\
\hline DOE131232_2 & 0 & 0 & 0 & 0 & 6 & 0 & 0 & 6 & 0 & 0 & 6 & 0 & 0 & 0 & 6 \\
\hline DOE 131232_3 & 3 & 4 & 3 & 2.19 & 161 & 3 & 4.05 & 313 & 14 & 4.41 & 331 & 20 & 0 & 10.17 & 300 \\
\hline DOE132231_1 & 3 & 4 & 3 & 0.5 & 66 & 0 & 5.36 & 618 & 30 & 7.01 & 658 & 29 & 0 & 11.14 & 722 \\
\hline DOE132231_2 & 1 & 2 & 1 & 4.72 & 366 & 5 & 8.94 & 685 & 27 & 12.28 & 835 & 29 & 0 & 26.66 & 1018 \\
\hline DOE132231_3 & 0 & 2 & 0 & 3.05 & 284 & 5 & 4.58 & 637 & 25 & 8.66 & 845 & 20 & 0 & 8.97 & 728 \\
\hline DOE132232_1 & 5 & 8 & 5 & 4.22 & 330 & 5 & 4.45 & 420 & 26 & 8.92 & 663 & 24 & 0 & 18.89 & 685 \\
\hline DOE132232_2 & 0 & 3 & 0 & 0.01 & 7 & 0 & 4.01 & 539 & 35 & 5.89 & 719 & 18 & 0 & 8.08 & 672 \\
\hline DOE132232_3 & 5 & 6 & 5 & 5.48 & 456 & 2 & 8.94 & 740 & 26 & 8.86 & 866 & 31 & 0 & 13.66 & 689 \\
\hline DOE133231_1 & 18 & - & 18 & 16.17 & 1136 & 7 & 42.33 & 2959 & 50 & 221.22 & 5228 & 152 & 1 & 91.09 & 2509 \\
\hline DOE133231_2 & 16 & - & 16 & 17.2 & 1124 & 7 & 38.7 & 2508 & 47 & 247.74 & 4938 & 172 & 1 & $>600$ & 7251 \\
\hline DOE133231_3 & 14 & - & 14 & 20.2 & 1413 & 7 & 50.58 & 3558 & 51 & 223.36 & 5524 & 112 & 0 & $>600$ & 8227 \\
\hline DOE 133232_1 & 26 & - & 26 & 26.56 & 1591 & 6 & 64.53 & 3980 & 41 & 128.35 & 3810 & 42 & 0 & $>600$ & 8558 \\
\hline DOE133232_2 & 38 & - & 38 & 24.72 & 1636 & 8 & 61.63 & 3892 & 47 & 195.52 & 5014 & 58 & 0 & 103.22 & 2228 \\
\hline DOE133232_3 & 44 & - & 44 & 29.59 & 1985 & 11 & 69.7 & 3861 & 47 & 71.5 & 2996 & 56 & 0 & 262.07 & 4916 \\
\hline DOE141231_1 & 0 & 0 & 0 & 0 & 7 & 0 & 0 & 7 & 0 & 0 & 7 & 0 & 0 & 0 & 7 \\
\hline DOE141231_2 & 0 & 0 & 0 & 0 & 7 & 0 & 0 & 7 & 0 & 0 & 7 & 0 & 0 & 0 & 7 \\
\hline DOE141231_3 & 0 & 0 & 0 & 0 & 7 & 0 & 0 & 7 & 0 & 0 & 7 & 0 & 0 & 0 & 7 \\
\hline DOE141232_1 & 0 & 0 & 0 & 0 & 7 & 0 & 0 & 7 & 0 & 0 & 7 & 0 & 0 & 0 & 7 \\
\hline DOE141232_2 & 0 & 0 & 0 & 0 & 7 & 0 & 0 & 7 & 0 & 0 & 7 & 0 & 0 & 0 & 7 \\
\hline DOE141232_3 & 1 & 1 & 1 & 4.77 & 95 & 0 & 0.84 & 77 & 0 & 0.88 & 77 & 0 & 0 & 1.69 & 77 \\
\hline DOE142231_1 & 2 & 2 & 2 & 65.42 & 659 & 6 & 3.64 & 310 & 0 & 3.08 & 271 & 0 & 0 & 5.59 & 271 \\
\hline DOE142231_2 & 5 & 5 & 5 & 24.95 & 305 & 0 & 2.73 & 235 & 0 & 3.05 & 244 & 0 & 0 & 5.81 & 244 \\
\hline DOE142231_3 & 2 & 4 & 2 & 68.41 & 740 & 8 & 54.78 & 1242 & 69 & 107.72 & 1870 & 61 & 0 & 497.53 & 2392 \\
\hline DOE142232_1 & 12 & 14 & 12 & 77.33 & 787 & 9 & 109.82 & 1401 & 63 & 175.47 & 1828 & 60 & 0 & 563.6 & 1285 \\
\hline DOE142232_2 & 2 & 4 & 2 & 57.65 & 639 & 7 & 94.09 & 1086 & 65 & 169.45 & 2264 & 70 & 1 & $>600$ & 1035 \\
\hline DOE142232_3 & 11 & 13 & 11 & 88.53 & 978 & 6 & 65.02 & 1257 & 59 & 113.76 & 1985 & 64 & 1 & $>600$ & 1480 \\
\hline DOE143231_1 & 6 & 10 & 6 & 218.26 & 2500 & 10 & 349.81 & 5015 & 74 & $>600$ & 6550 & 124 & 0 & $>600$ & 1477 \\
\hline DOE143231_2 & 8 & 9 & 8 & 140.03 & 1486 & 7 & 166.36 & 2363 & 84 & $>600$ & 5406 & 104 & 0 & $>600$ & 871 \\
\hline DOE143231_3 & 12 & 13 & 12 & 50.73 & 578 & 0 & 274.35 & 3151 & 81 & 245.24 & 1982 & 80 & 1 & $>600$ & 844 \\
\hline DOE143232_1 & 21 & 29 & 21 & 154.42 & 1411 & 7 & 349.31 & 4078 & 92 & $>600$ & 6103 & 159 & 0 & $>600$ & 912 \\
\hline DOE143232_2 & 16 & 26 & 16 & 150.01 & 1397 & 10 & 275.31 & 2341 & 81 & $>600$ & 4601 & 73 & 0 & $>600$ & 772 \\
\hline DOE143232_3 & 23 & 27 & 23 & 146.28 & 1271 & 7 & 315.14 & 4105 & 68 & $>600$ & 4064 & 73 & 0 & $>600$ & 858 \\
\hline DOE211231_1 & 5 & 5 & 5 & 0.05 & 8 & 0 & 0.06 & 10 & 0 & 0.05 & 10 & 0 & 0 & 0.05 & 10 \\
\hline DOE211231_2 & 5.5 & 6 & 6 & 0.06 & 12 & 0 & 0.03 & 7 & 0 & 0.03 & 7 & 0 & 0 & 0.05 & 7 \\
\hline DOE211231_3 & 4 & 4 & 4 & 0.06 & 10 & 0 & 0.06 & 11 & 0 & 0.06 & 11 & 0 & 0 & 0.06 & 11 \\
\hline DOE211232_1 & 8 & 8 & 8 & 0.03 & 6 & 0 & 0.03 & 6 & 0 & 0.03 & 6 & 0 & 0 & 0.03 & 6 \\
\hline DOE211232_2 & 5 & 12 & 5 & 0.17 & 31 & 0 & 0.16 & 33 & 0 & 0.17 & 33 & 0 & 0 & 0.17 & 33 \\
\hline DOE211232_3 & 11 & 21 & 11 & 0.11 & 20 & 0 & 0.11 & 22 & 0 & 0.11 & 22 & 0 & 0 & 0.11 & 22 \\
\hline DOE212231_1 & 5 & 13 & 5 & 0.25 & 50 & 1 & 0.33 & 70 & 2 & 0.33 & 69 & 3 & 0 & 0.38 & 80 \\
\hline DOE212231_2 & 7 & 8 & 8 & 0.48 & 94 & 3 & 0.61 & 124 & 4 & 0.61 & 120 & 4 & 0 & 0.64 & 122 \\
\hline DOE212231_3 & 8 & 12 & 8 & 0.38 & 81 & 1 & 0.33 & 78 & 2 & 0.34 & 74 & 3 & 0 & 0.36 & 79 \\
\hline DOE212232_1 & 24 & 29 & 25 & 0.89 & 160 & 8 & 0.75 & 152 & 5 & 0.69 & 142 & 2 & 0 & 0.95 & 184 \\
\hline DOE212232_2 & 20.5 & 24 & 21 & 0.28 & 58 & 2 & 0.25 & 58 & 1 & 0.28 & 64 & 2 & 0 & 0.3 & 63 \\
\hline DOE212232_3 & 24.33 & 36 & 25 & 0.34 & 77 & 0 & 0.45 & 90 & 4 & 0.52 & 105 & 4 & 1 & 0.48 & 89 \\
\hline DOE213231_1 & 13 & 14 & 13 & 0.7 & 165 & 0 & 0.94 & 208 & 1 & 0.94 & 208 & 1 & 0 & 0.95 & 205 \\
\hline DOE213231_2 & 13 & 15 & 13 & 0.67 & 154 & 1 & 0.8 & 172 & 4 & 0.81 & 172 & 5 & 0 & 0.97 & 196 \\
\hline DOE213231_3 & 13 & 15 & 13 & 1.06 & 237 & 1 & 0.83 & 186 & 0 & 1.03 & 232 & 1 & 0 & 1 & 223 \\
\hline DOE213232_1 & 41 & 44 & 41 & 0.63 & 143 & 1 & 1.24 & 248 & 6 & 0.75 & 160 & 2 & 0 & 1.08 & 213 \\
\hline DOE213232_2 & 39 & 44 & 39 & 0.5 & 111 & 2 & 0.75 & 156 & 3 & 0.88 & 183 & 5 & 0 & 1 & 196 \\
\hline DOE213232_3 & 45 & 47 & 45 & 1.03 & 211 & 4 & 0.52 & 121 & 2 & 0.81 & 171 & 4 & 0 & 0.66 & 145 \\
\hline DOE221231_1 & 1 & 1 & 1] & 0.16 & 28 & 0 & 0.17 & 33 & 0 & 0.16 & 32 & 0 & 0 & 0.17 & 32 \\
\hline DOE221231_2 & 2.17 & 3 & 3 & 0.75 & 86 & 0 & 0.66 & 99 & 0 & 0.47 & 75 & 0 & 0 & 0.53 & 75 \\
\hline DOE221231_3 & 0 & 1 & 0 & 0.38 & 50 & 1 & 0.3 & 48 & 1 & 0.56 & 82 & 3 & 0 & 0.67 & 90 \\
\hline DOE221232_1 & 0 & 0 & 0 & 0.41 & 50 & 1 & 0 & 4 & 0 & 0 & 4 & 0 & 0 & 0 & 4 \\
\hline DOE221232_2 & 1 & 1 & 1 & 0.17 & 24 & 0 & 0.2 & 40 & 0 & 0.22 & 38 & 0 & 0 & 0.23 & 38 \\
\hline DOE221232_3 & 0 & 0 & 0 & 0 & 4 & 0 & 0.02 & 4 & 0 & 0 & 4 & 0 & 0 & 0 & 4 \\
\hline
\end{tabular}




\begin{tabular}{|c|c|c|c|c|c|c|c|c|c|c|c|c|c|c|c|}
\hline & & & & $\begin{array}{r}\text { Branch } \\
\mathrm{v}\end{array}$ & $\begin{array}{l}\text { ing on } \\
\text { ariables }\end{array}$ & olumn & Branchi & $\begin{array}{l}\text { ng on ti } \\
\text { cells }\end{array}$ & letable & $\begin{array}{r}\text { Branc } \\
\text { prec }\end{array}$ & $\begin{array}{l}\text { hing on } \\
\text { cedence }\end{array}$ & $\begin{array}{l}\text { immedia } \\
\text { relations }\end{array}$ & & & $\begin{array}{l}\text { anching } \\
\text { ecedenc }\end{array}$ \\
\hline Problem & Root LP & Heur & Opt & Time & Cols & Nodes & Time & Cols & Nodes & Time & Cols & Nodes & Fract & Time & Cols \\
\hline DOE222231_1 & 6 & 11 & 6 & 17.31 & 1456 & 18 & 12.19 & 1089 & 10 & 22.03 & 1326 & 16 & 0 & 27.47 & 1445 \\
\hline DOE222231_2 & 5.82 & 12 & 7 & 147.08 & 3888 & 200 & 69.8 & 3325 & 86 & 76.03 & 3676 & 82 & 0 & 121.39 & 3829 \\
\hline DOE222231_3 & 6.42 & 10 & 7 & 36.25 & 2252 & 58 & 8.25 & 840 & 11 & 133.17 & 3774 & 86 & 0 & 449.3 & 6863 \\
\hline DOE222232_1 & 12.39 & 22 & 13 & 57.16 & 2824 & 74 & 25.98 & 1742 & 21 & 18.64 & 1197 & 15 & 0 & 71.73 & 2569 \\
\hline DOE222232_2 & 17 & 32 & 17 & 6.31 & 614 & 6 & 14.45 & 1273 & 13 & 7.51 & 672 & 3 & 0 & 16.42 & 1082 \\
\hline DOE222232_3 & 16 & 34 & 16 & 12.06 & 1007 & 7 & 7.22 & 700 & 4 & 5.19 & 495 & 2 & 0 & 8.55 & 642 \\
\hline DOE223231_1 & 13.09 & - & 14 & 41.61 & 3230 & 28 & $>600$ & 9976 & 191 & 71.52 & 3154 & 25 & 0 & $>600$ & 8596 \\
\hline DOE223231_2 & 13 & - & & $>600$ & 12231 & 286 & $>600$ & 11827 & 239 & $>600$ & 10656 & 148 & 0 & $>600$ & 9782 \\
\hline DOE223231_3 & 14.17 & 21 & 15 & 24.56 & 1826 & 14 & 435.92 & 8091 & 119 & 37.47 & 1868 & 14 & 0 & 41.08 & 1735 \\
\hline DOE223232_1 & 37 & - & 37 & 12.95 & 1070 & 6 & 32.45 & 2006 & 15 & 38.94 & 1792 & 14 & 0 & 51.66 & 1980 \\
\hline DOE223232_2 & 39.14 & - & & $>600$ & 8328 & 189 & $>600$ & 9718 & 141 & $>600$ & 8950 & 126 & 0 & $>600$ & 8583 \\
\hline DOE223232_3 & 29 & 77 & - & $>600$ & 13427 & 234 & $>600$ & 12784 & 171 & $>600$ & 10899 & 82 & 0 & $>600$ & 11191 \\
\hline DOE231231_1 & 0 & 0 & 0 & 1.77 & 141 & 3 & 0 & 6 & 0 & 0 & 6 & 0 & 0 & 0 & 6 \\
\hline DOE231231_2 & 3 & 3 & 3 & 1.2 & 119 & 0 & 0.64 & 95 & 0 & 0.53 & 83 & 0 & 이 & 0.59 & 83 \\
\hline DOE231231_3 & 1 & 2 & 1 & 3.88 & 250 & 3 & 3.06 & 251 & 10 & 5.19 & 415 & 15 & 0 & 5.25 & 227 \\
\hline DOE231232_1 & 3 & 5 & 3 & 3.28 & 198 & 4 & 4.42 & 319 & 10 & 3.64 & 247 & 11 & 0 & 6.23 & 245 \\
\hline DOE231232_2 & 0 & 1 & 0 & 2.34 & 172 & 6 & 2.23 & 265 & 15 & 3.34 & 364 & 15 & 0 & 4.39 & 400 \\
\hline DOE231232_3 & 0 & 4 & 0 & 2.03 & 172 & 3 & 3.05 & 362 & 12 & 3.34 & 362 & 13 & 0 & 4.08 & 350 \\
\hline DOE232231_1 & 3 & 6 & 3 & 11.95 & 699 & 9 & 9.36 & 793 & 17 & 43.83 & 2123 & 35 & 0 & 27.78 & 983 \\
\hline DOE232231_2 & 1 & 5 & 1 & 15.69 & 904 & 4 & 58.59 & 2606 & 31 & 15.5 & 809 & 9 & o & 33.14 & 1075 \\
\hline DOE232231_3 & 6 & 10 & 6 & 7.95 & 470 & 4 & 18.34 & 1034 & 17 & 23.28 & 1068 & 18 & 0 & 27.44 & 792 \\
\hline DOE232232_1 & 5 & 12 & 5 & 19.5 & 1177 & 7 & 32.11 & 1865 & 25 & 31.36 & 1612 & 30 & 0 & 11.61 & 613 \\
\hline DOE232232_2 & 11 & 18 & 11 & 10.2 & 491 & 4 & 11.73 & 803 & 8 & 14.27 & 832 & 15 & 0 & 35.02 & 1125 \\
\hline DOE232232_3 & 2 & 9 & 2 & 11.31 & 681 & 5 & 6.84 & 576 & 13 & 16.34 & 925 & 13 & 0 & 21.58 & 834 \\
\hline DOE233231_1 & 18 & - & - & $>600$ & 11742 & 136 & $>600$ & 9396 & 66 & $>600$ & 5875 & 29 & 0 & $>600$ & 5329 \\
\hline DOE233231_2 & 19 & - & & $>600$ & 12674 & 199 & $>600$ & 8739 & 36 & $>600$ & 5818 & 25 & c) & $>600$ & 6123 \\
\hline DOE233231_3 & 20 & - & - & $>600$ & 15023 & 171 & 602.1 & 8948 & 48 & $>600$ & 5440 & 18 & 이 & 494.19 & 5056 \\
\hline DOE233232_1 & 41 & - & - & $>600$ & 13107 & 99 & 601.2 & 7883 & 20 & $>600$ & 6007 & 11 & 0 & $>600$ & 4680 \\
\hline DOE233232_2 & 52 & - & 52 & 81.61 & 2950 & 10 & 242.16 & 4867 & 22 & $>600$ & 5923 & 27 & 0 & $>600$ & 5334 \\
\hline DOE233232_3 & 56 & - & - & $>600$ & 14705 & 216 & $>600$ & 9205 & 88 & $>600$ & 6871 & 30 & 0 & $>600$ & 6237 \\
\hline DOE241231_1 & 0 & 0 & 0 & 0.02 & 7 & 0 & 0 & 7 & 0 & 0 & 7 & 0 & 0 & 0 & 7 \\
\hline DOE241231_2 & 0 & 0 & 0 & 0 & 7 & 0 & 0 & 7 & 0 & 0.02 & 7 & 0 & 0 & 0 & 7 \\
\hline DOE241231_3 & 0 & 0 & 0 & 0.02 & 7 & 0 & 0 & 7 & 0 & 0 & 7 & 0 & 0 & 0 & 7 \\
\hline DOE241232_1 & 0 & 0 & 0 & 0 & 7 & 0 & 0 & 7 & 0 & 0.01 & 7 & 0 & 0 & 0 & 7 \\
\hline DOE241232_2 & 0 & 0 & 0 & 21.86 & 248 & 5 & 0 & 7 & 0 & 0 & 7 & 0 & 0 & 0 & 7 \\
\hline DOE241232_3 & 0 & 0 & 0 & 0.01 & 7 & 0 & 0 & 7 & 0 & 0 & 7 & 0 & 0 & 0.02 & 7 \\
\hline DOE242231_1 & 4 & 9 & 4 & 244.51 & 2376 & 14 & $>600$ & 4877 & 75 & 432.5 & 3005 & 41 & 0 & $>600$ & 1096 \\
\hline DOE242231_2 & 0 & 4 & 0 & 81.16 & 1089 & 9 & 226.99 & 4527 & 86 & $>600$ & 5294 & 44 & 0 & $>600$ & 3755 \\
\hline DOE242231_3 & 2 & 6 & - & $>600$ & 4575 & 341 & $>600$ & 7025 & 72 & $>600$ & 3711 & 39 & 0 & $>600$ & 1143 \\
\hline DOE2422.32_1 & 2 & 15 & 2 & 159.63 & 1694 & 27 & $>600$ & 6241 & 118 & 583 & 4695 & 40 & 0 & $>600$ & 2222 \\
\hline DOE242232_2 & 3 & 20 & 3 & 173.55 & 2135 & 11 & $>600$ & 8812 & 102 & 183.64 & 2694 & 32 & 0 & $>600$ & 4003 \\
\hline DOE242232_3 & 1 & 15 & 1 & 288.18 & 2795 & 11 & 362.04 & 4606 & 45 & $>600$ & 3867 & 37 & 이 & $>600$ & 1471 \\
\hline DOE243231_1 & 9 & 19 & - & $>600$ & 5310 & 33 & $>600$ & 4308 & 34 & $>600$ & 2276 & 27 & 0 & $>600$ & 973 \\
\hline DOE243231_2 & 8 & - & - & $>600$ & 3986 & 6 & $>600$ & 3506 & 20 & $>600$ & 2011 & 21 & 0 & $>600$ & 913 \\
\hline DOE243231_3 & 6 & - & - & $>600$ & 4178 & 4 & $>600$ & 4652 & 25 & $>600$ & 2529 & 28 & 0 & $>600$ & 1215 \\
\hline DOE243232_1 & 19 & - & - & $>600$ & 3551 & 8 & $>600$ & 3450 & 10 & $>600$ & 2115 & 15 & 0 & $>600$ & 1134 \\
\hline DOE243232_2 & 20 & 43 & & $>600$ & 3445 & 4 & $>600$ & 4772 & 24 & $>600$ & 2129 & 19 & 0 & $>600$ & 1142 \\
\hline DOE243232_3 & 30 & 68 & 30 & 479.74 & 3195 & 8 & $>600$ & 3197 & 13 & $>600$ & 1992 & 18 & 0 & $>600$ & 1042 \\
\hline DOE311231_1 & 9 & 9 & 9 & 0.06 & 9 & 0 & 0.11 & 17 & 0 & 0.13 & 17 & 0 & 0 & 0.13 & 17 \\
\hline DOE311231_2 & 6 & 6 & 6 & 0.06 & 10 & 0 & 0.05 & 9 & 0 & 0.05 & 9 & 0 & 0 & 0.06 & 9 \\
\hline DOE311231_3 & 4 & 4 & 4 & 0.13 & 13 & 이 & 0.09 & 12 & 0 & 0.08 & 12 & 0 & 0 & 0.09 & 12 \\
\hline DOE311232_1 & 23 & 23 & 23 & 0.06 & 8 & 0 & 0.08 & 11 & 0 & 0.06 & 11 & 0 & 0 & 0.06 & 11 \\
\hline DOE311232_2 & 12 & 12 & 12 & 0.09 & 14 & 이 & 0.05 & 7 & 0 & 0.05 & 7 & 0 & 0 & 0.03 & 7 \\
\hline DOE311232_3 & 14 & 14 & 14 & 0.08 & 13 & 0 & 0.08 & 14 & 0 & 0.09 & 14 & 0 & 0 & 0.08 & 14 \\
\hline DOE312231_1 & 11 & 12 & 11 & 0.08 & 14 & 0 & 0.38 & 50 & 3 & 0.25 & 35 & 1 & 0 & 0.25 & 35 \\
\hline DOE312231_2 & 13 & 17 & 13 & 0.5 & 76 & 1 & 0.39 & 63 & 0 & 0.38 & 63 & 0 & 0 & 0.39 & 63 \\
\hline DOE312231_3 & 11 & 12 & 11 & 0.14 & 24 & 0 & 0.27 & 43 & 1 & 0.3 & 42 & 1 & 이 $1-1$ & 0.28 & 42 \\
\hline DOE312232_1 & 28 & 33 & 28 & 0.33 & 47 & 1 & 0.39 & 58 & 1 & 0.33 & 50 & 1 & 0 & 0.34 & 53 \\
\hline DOE312232_2 & 30 & 32 & 30 & 0.48 & 71 & 3 & 0.33 & 51 & 0 & 0.33 & 51 & 0 & 0 & 0.33 & 51 \\
\hline DOE312232_3 & 33 & 33 & 33 & 0.14 & 23 & 0 & 0.09 & 19 & 0 & 0.11 & 19 & 0 & 0 & 0.09 & 19 \\
\hline DOE313231_1 & 18.36 & - & 19 & 0.75 & 133 & 0 & 1.36 & 214 & 7 & 1.44 & 226 & 5 & 0 & 1.72 & 255 \\
\hline DOE313231_2 & 19.5 & 20 & 20 & 0.53 & 93 & 0 & 0.44 & 81 & 0 & 0.44 & 81 & 0 & 0 & 0.45 & 81 \\
\hline DOE313231_3 & 18 & - & 18 & 1.13 & 175 & 4 & 0.64 & 107 & 1 & 0.63 & 104 & 1 & 0 & 0.63 & 104 \\
\hline DOE313232_1 & 53 & - & 53 & 0.88 & 142 & 2 & 0.84 & 131 & 3 & 1.44 & 219 & 5 & 0 & 1.09 & 169 \\
\hline DOE313232_2 & 51 & 55 & 51 & 1.11 & 175 & 2 & 1.28 & 207 & 2 & 1.56 & 234 & 4 & 0 & 1.86 & 272 \\
\hline DOE313232_3 & 55 & - & 55 & 0.92 & 157 & 1 & 0.89 & 155 & 0 & 0.89 & 155 & 0 & 0 & 0.91 & 155 \\
\hline DOE321231_1 & 2 & 3 & 2 & 1.31 & 95 & 1 & 1.2 & 127 & 1 & 0.69 & 74 & 1 & 0 & 0.73 & 74 \\
\hline DOE321231_2 & 1 & 2 & 1 & 0.83 & 73 & 1 & 2.94 & 231 & 12 & 4.99 & 374 & 18 & 0 & 5.75 & 410 \\
\hline DOE321231_3 & 1 & 1 & 1 & 0.72 & 55 & 0 & 0.2 & 28 & 0 & 0.2 & 28 & 0 & 0 & 0.22 & 28 \\
\hline DOE321232_1 & 1.21 & 4 & 2 & 1.42 & 105 & 0 & 1.83 & 179 & 8 & 1.78 & 172 & 5 & 0 & 1.28 & 116 \\
\hline DOE321232_2 & 4.33 & 7 & 5 & 1.13 & 78 & 1 & 1.13 & 106 & 1 & 2.02 & 168 & 5 & 0 & 3.09 & 222 \\
\hline DOE321232_3 & 10.29 & 13 & 11 & 3.81 & 231 & 7 & 2.48 & 220 & 9 & 1.58 & 150 & 5 & 0) & 4.2 & 307 \\
\hline DOE322231_1 & 8.15 & 15 & 9 & 29.24 & 1609 & 21 & 9.63 & 650 & 4 & 31.39 & 1574 & 16 & 이 & 90.04 & 3065 \\
\hline DOE322231_2 & 14 & 18 & 14 & 5.49 & 353 & 0 & 4.56 & 338 & 0 & 4.66 & 341 & 0 & 0 & 5.63 & 341 \\
\hline DOE322231_3 & 9.34 & 16 & 10 & 26.05 & 1351 & 67 & 9.94 & 664 & 11 & 11.47 & 718 & 10 & 0 & 12.42 & 660 \\
\hline DOE322232_1 & 23 & 51 & 23 & 6.25 & 407 & 1 & 6.8 & 502 & 4 & 6.41 & 470 & 5 & 0 & 5.95 & 380 \\
\hline DOE322232_2 & 20 & 44 & 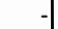 & $>600$ & 13527 & 488 & $>600$ & 11901 & 366 & 302.46 & 6115 & 102 & 0 & $>600$ & 9358 \\
\hline DOE322232_3 & 22.8 & 40 & 25 & 515.91 & 9512 & 341 & 345.18 & 7984 & 224 & 255.94 & 5362 & 180 & 0 & 326.94 & 6457 \\
\hline DOE323231_1 & 22 & - & 22 & 18.75 & 1003 & 5 & 44.33 & 2118 & 15 & 51.3 & 1868 & 13 & 0 & 83.17 & 2142 \\
\hline DOE323231_2 & 24 & - & 24 & 15.98 & 923 & 6 & 23.62 & 1379 & 24 & 31.75 & 1247 & 15 & 0 & 47.72 & 1651 \\
\hline DOE323231_3 & 23 & 30 & 23 & 267.89 & 7368 & 108 & 158.56 & 4388 & 41 & 177.7 & 3876 & 19 & 0 & 331.77 & 5281 \\
\hline
\end{tabular}




\begin{tabular}{|c|c|c|c|c|c|c|c|c|c|c|c|c|c|c|c|c|c|}
\hline \multirow[b]{2}{*}{ Problem } & \multirow[b]{2}{*}{ Root LP } & \multirow[b]{2}{*}{ Heur } & \multirow[b]{2}{*}{ Opt } & \multicolumn{3}{|c|}{$\begin{array}{c}\text { Branching on column } \\
\text { variables }\end{array}$} & \multicolumn{3}{|c|}{$\begin{array}{c}\text { Branching on timetable } \\
\text { cells }\end{array}$} & \multicolumn{4}{|c|}{$\begin{array}{l}\text { Branching on immediate } \\
\text { precedence relations }\end{array}$} & \multicolumn{4}{|c|}{$\begin{array}{l}\text { Branching on normal } \\
\text { precedence relations }\end{array}$} \\
\hline & & & & Time & Cols & Nodes & Time & Cols & Nodes & Time & Cols & Nodes & Fract & Time & Cols & Nodes & Fract \\
\hline DOE323232_1 & 61 & & 61 & 14.02 & 793 & 5 & 30.83 & 1514 & 13 & 56.94 & 2098 & 15 & 0 & 50.97 & 1874 & 28 & 0 \\
\hline DOE323232_2 & 63 & - & 63 & 15.11 & 871 & 4 & 47.91 & 2039 & 14 & 66.44 & 2051 & 11 & 0 & 76.61 & 1966 & 18 & 0 \\
\hline DOE323232_3 & 53 & - & 53 & 58.02 & 2877 & 24 & 61.31 & 2447 & 13 & 77.78 & 2073 & 8 & 0 & $>600$ & 6628 & 53 & 0 \\
\hline DOE331231_1 & 4 & 7 & 4 & 7.27 & 272 & 4 & 11.17 & 491 & 11 & 8.31 & 346 & 8 & 0 & 15.41 & 467 & 22 & 0 \\
\hline DOE331231_2 & 0 & 3 & 0 & 9.48 & 470 & 4 & 8.83 & 543 & 3 & 14.84 & 524 & 2 & 0 & 28.36 & 717 & 4 & 0 \\
\hline DOE331231_3 & 4 & 7 & 4 & 50.55 & 1237 & 34 & 14.27 & 681 & 11 & 13.3 & 600 & 10 & 0 & 9.45 & 389 & 7 & 0 \\
\hline DOE331232_1 & 4 & 8 & 4 & 171.14 & 2505 & 191 & 23.63 & 1184 & 18 & 7.42 & 402 & 7 & 0 & 21.27 & 919 & 13 & 0 \\
\hline DOE331232_2 & 1 & 9 & 1 & 10.56 & 500 & 2 & 8.64 & 588 & 7 & 7.09 & 471 & 6 & 0 & 10.64 & 560 & 10 & 0 \\
\hline DOE331232_3 & 6 & 15 & 7 & $>600$ & 6563 & 422 & 84.44 & 2626 & 58 & 383.95 & 6809 & 227 & 0 & $>600$ & 8228 & 348 & 0 \\
\hline DOE332231_1 & 9 & 14 & 9 & 172.48 & 3395 & 66 & 345.11 & 5089 & 78 & 597.3 & 6078 & 88 & 0 & $>600$ & 5541 & 88 & 0 \\
\hline DOE332231_2 & 9 & 12 & 9 & 54.42 & 1453 & 8 & 33.42 & 1139 & 14 & 35.39 & 826 & 8 & 0 & 60.31 & 998 & 12 & 0 \\
\hline DOE332231_3 & 5 & 10 & 5 & $>600$ & 6504 & 377 & 358.31 & 6260 & 154 & 143.86 & 2958 & 36 & 0 & 153.4 & 2906 & 53 & 0 \\
\hline DOE332232_1 & 20 & 35 & 20 & 251.77 & 3725 & 135 & 77.66 & 2055 & 15 & 58.08 & 1228 & 11 & 0 & 50.18 & 969 & 17 & 0 \\
\hline DOE332232_2 & 21 & 37 & 21 & 36.42 & 1136 & 7 & 40.67 & 1524 & 13 & 350.62 & 4744 & 46 & 0 & 63.46 & 1410 & 22 & 0 \\
\hline DOE332232_3 & 9 & 24 & 9 & $>600$ & 6706 & 659 & 27.89 & 1175 & 8 & $>600$ & 6146 & 107 & 0 & $>600$ & 5770 & 114 & 0 \\
\hline DOE333231_1 & 27 & - & & 598.6 & 9247 & 27 & $>600$ & 7268 & 30 & $>600$ & 4769 & 16 & 이 & $>600$ & 4443 & 27 & 0 \\
\hline DOE333231_2 & 28 & - & 28 & 440.78 & 8727 & 106 & $>600$ & 8012 & 31 & $>600$ & 4275 & 15 & 0 & $>600$ & 4404 & 29 & 0 \\
\hline DOE333231_3 & 27 & - & - & $>600$ & 8807 & 86 & $>600$ & 6648 & 23 & $>600$ & 5259 & 26 & 0 & $>600$ & 3775 & 46 & 0 \\
\hline DOE333232_1 & 68 & - & & $>600$ & 6247 & 4 & $>600$ & 4423 & 5 & $>600$ & 4102 & 4 & 0 & $>600$ & 4003 & 4 & 0 \\
\hline DOE333232_2 & 75 & - & - & $>600$ & 9474 & 6 & $>600$ & 5398 & 5 & $>600$ & 4724 & 4 & 0 & $>600$ & 4358 & 4 & 0 \\
\hline DOE333232_3 & 68.08 & - & & $>600$ & 7545 & 8 & $>600$ & 5056 & 6 & $>600$ & 5125 & 7 & 0 & $>600$ & 4649 & 7 & 0 \\
\hline DOE341231_1 & 1 & 2 & 1 & 46.86 & 489 & 6 & 68.54 & 820 & 34 & 105.35 & 973 & 26 & 이 & 347.27 & 1428 & 64 & 0 \\
\hline DOE341231_2 & 2 & 2 & 2 & 12.36 & 132 & 0 & 3.72 & 186 & 0 & 4.61 & 166 & 0 & 0 & 6.75 & 166 & 0 & 0 \\
\hline DOE341231_3 & 1 & 1 & 1 & 9.14 & 130 & 0 & 2.59 & 151 & 0 & 2.27 & 148 & 0 & 이 & 2.67 & 148 & 0 & 0 \\
\hline DOE341232_1 & 0 & 3 & 0 & 0 & 7 & 0 & 14.71 & 631 & 35 & 28.78 & 1014 & 25 & 0 & 72.65 & 1477 & 67 & 0 \\
\hline DOE341232_2 & 0 & 2 & 0 & 33.93 & 330 & 7 & 46.26 & 808 & 38 & 73.93 & 1105 & 23 & 0 & 129.48 & 932 & 65 & 0 \\
\hline DOE341232_3 & 0 & 0 & 0 & 0 & 7 & 0 & 0 & 7 & 0 & 0 & 7 & 0 & 이 & 0 & 7 & 0 & 0 \\
\hline DOE342231_1 & 4 & 10 & - & $>600$ & 4225 & 25 & $>600$ & 3914 & 40 & $>600$ & 1919 & 15 & 0 & $>600$ & 1211 & 40 & 0 \\
\hline DOE342231_2 & 4 & 15 & & $>600$ & 3401 & 10 & $>600$ & 3153 & 17 & $>600$ & 1815 & 15 & 0 & $>600$ & 1060 & 13 & 0 \\
\hline DOE342231_3 & 7 & 14 & & $>600$ & 3740 & 55 & $>600$ & 3334 & 20 & $>600$ & 1969 & 26 & 0 & $>600$ & 989 & 27 & 0 \\
\hline DOE342232_1 & 4 & 38 & & $>600$ & 3013 & 6 & $>600$ & 3397 & 10 & $>600$ & 1973 & 9 & 0 & $>600$ & 1191 & 12 & 0 \\
\hline DOE342232_2 & 7 & 44 & 7 & 431.45 & 2403 & 5 & $>600$ & 4587 & 25 & $>600$ & 2608 & 18 & 0 & $>600$ & 1406 & 32 & 0 \\
\hline DOE342232_3 & 6 & 10 & 1 & $>600$ & 3895 & 28 & $>600$ & 3527 & 32 & $>600$ & 2050 & 21 & 0 & $>600$ & 984 & 33 & 0 \\
\hline DOE343231_1 & 13.78 & 30 & & $>600$ & 1510 & 0 & $>600$ & 1805 & 0 & $>600$ & 1695 & 0 & 0 & $>600$ & 1105 & 0 & 0 \\
\hline DOE343231_2 & 11 & 34 & & $>600$ & 2235 & 1 & $>600$ & 2581 & 5 & $>600$ & 1666 & 5 & 0 & $>600$ & 1145 & 1 & 0 \\
\hline DOE343231_3 & 13.17 & - & & $>600$ & 1460 & 0 & $>600$ & 1590 & 0 & $>600$ & 1546 & 0 & 0 & $>600$ & 956 & 0 & 0 \\
\hline DOE343232_1 & 26.45 & - & - & $>600$ & 1360 & 0 & $>600$ & 1710 & 0 & $>600$ & 1687 & 0 & 0 & $>600$ & 1107 & 0 & 0 \\
\hline DOE343232_2 & 26.37 & - & & $>600$ & 1320 & 0 & $>600$ & 1607 & 0 & $>600$ & 1584 & 0 & 0 & $>600$ & 1024 & 0 & 0 \\
\hline DOE343232_3 & 31.62 & 97 & - & $>600$ & 1410 & 0 & $>600$ & 2334 & 4 & $>600$ & 1928 & 2 & 0 & $>600$ & 1318 & 0 & 0 \\
\hline
\end{tabular}

